\title{
ERK Regulates NeuroD1-mediated Neurite Outgrowth via Proteasomal Degradation
}

\author{
Tae-young Lee ${ }^{1,2,3}$, In-Su Cho $^{1}$, Narayan Bashyal ${ }^{1,2}$, Francisco J Naya ${ }^{4}$, Ming-Jer Tsai ${ }^{5}$, \\ Jeong Seon Yoon ${ }^{1}$, Jung-Mi Choi ${ }^{1}$, Chang-Hwan Park ${ }^{6}$, Sung-Soo Kim ${ }^{1,2 *}$ and Haeyoung Suh-Kim ${ }^{1,2,3 *}$ \\ ${ }^{1}$ Department of Anatomy, Ajou University School of Medicine, Suwon 16499, ${ }^{2}$ Department of Biomedical Sciences, Graduate \\ School, Ajou University School of Medicine, Suwon 16499, ${ }^{3}$ Research Center, CelleBrain Ltd., Jeonju 54871, Korea, \\ ${ }^{4}$ Department of Biology, Life Science and Engineering Building, Boston University, Boston, MA 00215, ${ }^{5}$ Department of \\ Medicine and Program in Developmental Biology, Baylor College of Medicine, Houston, TX 77030, USA, ${ }^{6}$ Graduate School of \\ Biomedical Science and Engineering, Hanyang University, Seoul 04763, Korea
}

\begin{abstract}
Neurogenic differentiation 1 (NeuroD1) is a class B basic helix-loop-helix (bHLH) transcription factor and regulates differentiation and survival of neuronal and endocrine cells by means of several protein kinases, including extracellular signal-regulated kinase (ERK). However, the effect of phosphorylation on the functions of NeuroD1 by ERK has sparked controversy based on context-dependent differences across diverse species and cell types. Here, we evidenced that ERK-dependent phosphorylation controlled the stability of NeuroD1 and consequently, regulated proneural activity in neuronal cells. A null mutation at the ERK-dependent phosphorylation site, S274A, increased the half-life of NeuroD1 by blocking its ubiquitin-dependent proteasomal degradation. The S274A mutation did not interfere with either the nuclear translocation of NeuroD1 or its heterodimerization with E47, its ubiquitous partner and class A bHLH transcription factor. However, the S274A mutant increased transactivation of the E-box-mediated gene and neurite outgrowth in F11 neuroblastoma cells, compared to the wild-type NeuroD1. Transcriptome and Gene Ontology enrichment analyses indicated that genes involved in axonogenesis and dendrite development were downregulated in NeuroD1 knockout (KO) mice. Overexpression of the S274A mutant salvaged neurite outgrowth in NeuroD1-deficient mice, whereas neurite outgrowth was minimal with S274D, a phosphomimicking mutant. Our data indicated that a longer protein half-life enhanced the overall activity of NeuroD1 in stimulating downstream genes and neuronal differentiation. We propose that blocking ubiquitin-dependent proteasomal degradation may serve as a strategy to promote neuronal activity by stimulating the expression of neuron-specific genes in differentiating neurons.
\end{abstract}

Key words: Neurogenic differentiation factor 1, Neurite outgrowth, Extracellular signal-regulated kinase, Phosphorylation

\section{INTRODUCTION}

Neurogenic differentiation factor 1 (NeuroD1) is a tissue-specific (class B) member of the basic helix-loop-helix (bHLH) protein

Submitted June 1,2020, Revised June 16,2020,

Accepted June 16, 2020

* To whom correspondence should be addressed.

Sung-Soo Kim, TEL: 82-31-219-5036, FAX: 82-31-219-5034

e-mail:kimdmg@ajou.ac.kr

Haeyoung Suh-Kim, TEL: 82-31-219-5036, FAX: 82-31-219-5039

e-mail:hysuh@ajou.ac.kr family and plays a critical role in the commitment of neuronal precursors to neuronal differentiation [1]. NeuroD1 was first identified as a neurogenic transcription factor that could induce cell fate change after it was observed that presumptive epidermal cells could develop into fully differentiated neurons in Xenopus embryos [2]. In mice, NeuroD1 is highly expressed in several regions of the developing nervous system, as well as in adult neurons [24]. The targeted mutation of NeuroD1 induces a dramatic loss of neurons, due to extensive cell death, in the cerebellum, hippocampal dentate gyrus, and the inner ear [5-7]. Knockdown of NeuroD1 in primary cerebellar granule neurons dramatically reduces dendritic growth and arborization without altering axonal growth.
Copyright (c) Experimental Neurobiology 2020. www.enjournal.org
This is an Open Access article distributed under the terms of the Creative Commons Attribution Non-Commercial License (http://creativecommons.org/licenses/by-nc/4.0) which permits unrestricted non-commercial use, distribution, and reproduction in any medium, provided the original work is properly cited. 
These findings indicate that NeuroD1 is essential for the survival and maturation of adult-born neurons, particularly with regard to dendrite morphogenesis.

NeuroD1 (also known as NeuroD and BETA2) is expressed in developing enteroendocrine cells of the pancreas and intestine and plays a critical role in the differentiation and survival of these cells [8-10]. NeuroD1 forms heterodimers with class A bHLH partners, such as E47 and induces cell-type-specific gene expression in mature neuroendocrine cells. NeuroD1 also induces insulin and secretin gene expression in pancreatic $\beta$-cells and intestinal enteroendocrine cells, respectively $[9,11,12]$. Therefore, the global deletion of NeuroD1 causes neonatal diabetes, whereas the overexpression of NeuroD1 together with pancreatic and duodenal homeobox 1 (PDX1) and MAF BZIP Transcription Factor A (MafA) converts hepatocytes or intestinal cells into insulin-secreting cells [13-16].

Extracellular signal-regulated kinases 1/2 (ERK1/2) appear to integrate long- and short-term nutrient sensing information in the nucleus of $\beta$-cells to maintain insulin homeostasis. The modulation of NeuroD1 by ERK1/2 has been studied extensively in insulinoma cell lines, such as $\beta$ TC, INS-1, and MIN6 cells. Glucose induces ERK-dependent phosphorylation of four Ser residues, namely S162, S259, S266, and S274, in NeuroD1 in a $\mathrm{Ca}^{2+}$ dependent manner [17]. Among Ser to Ala mutations at these sites (S162A, S259A, S266A, and S274A), S274A exerts the most prominent effect [17]. Indeed, S274 is involved in the nuclear translocation of NeuroD1 in response to stimulating glucose levels [18]. The abovementioned mutations decrease promoter activity of the insulin gene when tested as chimeric proteins with GAL4 DNAbinding domain. In contrast to what was observed in insulinoma cell lines, S162A, S259A, S266A, and S274A mutants of Xenopus NeuroD1 (xNeuroD1) are significantly more capable of forming ectopic neurons [19]. In addition, S266A and S274A resulted in accumulation of $\mathrm{xNeuroD}$ protein in the injected embryos. Despite the contradicting findings of the interactions between ERK1/2 and NeuroD1, the mechanism of context-dependent NeuroD1 activity has remained inconclusive.

In this study, we investigated the molecular mechanisms underlying the context-dependent activity of NeuroD1 in neuronal cells with respect to its ubiquitin-dependent proteolysis, nuclear localization, and heterodimer formation with E47, and the transactivation of target genes. We propose that the ERK-dependent phosphorylation of NeuroD1 may be a key mechanism for maintaining neural progenitor pools during early brain development and for switching the neurogenic-to-gliogenic competence in neural progenitor cells during late brain development. Additionally, we suggest that blocking ubiquitin-dependent proteasomal degrada- tion, thereby stimulating the expression of neuron-specific genes during neuronal differentiation, may be used to promote neuronal activity.

\section{MATERIALS AND METHODS}

\section{Animals}

All experimental procedures using animals were approved by the Institutional Animal Care and Use Committee (IACUC) of Ajou University School of Medicine, South Korea (ethics number: 20150043). NeuroD $1^{+ \text {LacZ }}$ mice were interbred to obtain NeuroDI $1^{+/+}$ and NeuroD $I^{\text {LacZ/LacZ }}$ littermates [8]. Genotypes were determined by PCR-based analysis of genomic tail DNA using primers NeuroD1-F (5'-CTT GAA GCC ATG AAT GCA-3') and NeuroD1R (5'-TGA CAG AGC CCA GAT GTA-3') for the wild type (WT) allele and B2.lacZ (5'-ATC GAT CTC GCC ATA CAG-3') for the mutant. The mice were housed in groups of $3 \sim 4$ per cage, with ad libitum access to food and water, and maintained in a 12:12 h light-dark cycle. All mice were anesthetized with 2,2,2-Tribromoethanol (Sigma-Aldrich, T48402) $(250 \mathrm{mg} / \mathrm{kg})$ and euthanized by $\mathrm{CO}_{2}$ asphyxiation.

\section{Cell culture and transfection}

HEK 293T and F11 cells were maintained in Dulbecco's modified Eagle's medium (DMEM) supplemented with 10\% fetal bovine serum (FBS), $100 \mathrm{units} / \mathrm{ml}$ penicillin, and $100 \mu \mathrm{g} / \mathrm{ml}$ streptomycin and incubated in a humidified $5 \% \mathrm{CO}_{2}$ incubator at $37^{\circ} \mathrm{C}$. Cell lines with a maximum of ten passages were used. Cells were transiently transfected using polyethyleneimine (PEI; Polysciences, 23966) as a DNA carrier. To measure the half-life of NeuroD1, 100 $\mu \mathrm{g} / \mathrm{ml}$ cycloheximide (Sigma-Aldrich, C4859) was added to the culture at the 0,30,60 and 120 min before harvest. Furthermore, $20 \mu \mathrm{M}$ PD 98059 (Sigma-Aldrich, P215) or $10 \mu \mathrm{M}$ N-acetyl-LeuLeu-norleucinal (ALLN) (Sigma-Aldrich, A6185) was added to the cell culture $30 \mathrm{~min}$ or $2 \mathrm{~h}$ prior to harvesting to inhibit the ERK or proteasome pathway, respectively.

\section{Preparation of lentiviral vectors}

Lentiviral transfer vectors, encoding the Flag-tagged WT and mutant NeuroD1 together with green fluorescent protein (GFP), were generated by replacing the hepatocyte growth factor (HGF) cDNA in pLenti-GIII-CMV-GFP-2A-Puro (Abm, LV 180162) with the corresponding cDNA from the abovementioned proteins. Using PEI, HEK 293T cells $\left(2 \times 10^{6}\right.$ cells in a $150-\mathrm{mm}^{2}$ dish $)$ were transfected with $7.5 \mu \mathrm{g}$ of each transfer plasmid; $5 \mu \mathrm{g}$ of each packaging plasmid, pPACKH1-GAG and pPACKH1-REV; and $2.5 \mu \mathrm{g}$ of pVSV-G (Systembio, LV500A-1). Thereafter, the con- 
ditioned medium was harvested twice, after 40 and $60 \mathrm{~h}$, placed in a tabletop centrifuge with $10 \%$ sucrose-containing buffer, and centrifuged at 14,000 $g$ overnight, following the method described previously [20].

\section{Subcellular fractionation and western blotting analysis}

HEK 293 T cells cells $\left(1 \times 10^{6}\right.$ cells in a $100-\mathrm{mm}^{2}$ dish $)$ were transfected with NeuroD1 expression vectors and cultured to confluence. The cell lysate-enriched cytosolic fraction was prepared by scraping the cells into RIPA Lysis and Extraction Buffer (Thermo Scientific, 89901) containing Protease Inhibitor Cocktail (SigmaAldrich, P2714). Subcellular fractionation was performed using the Subcellular Protein Fractionation Kit (Thermo Scientific, 78840), according to the manufacturer's instructions. Protein concentration was determined using the Pierce ${ }^{\mathrm{TM}}$ bicinchoninic acid (BCA) Protein Assay Kit (Thermo Fisher Scientific, 23225). Proteins were separated by sodium dodecyl sulfate polyacrylamide gel electrophoresis (SDS-PAGE) using 8\% or 10\% gel and transferred onto Amersham ${ }^{\mathrm{TM}}$ Hybond $^{\mathrm{TM}} 0.2-\mu \mathrm{m}$ polyvinylidene difluoride (PVDF) membranes (GE Healthcare, 1060021). The primary antibodies used were Flag epitope (Sigma-Aldrich, F1804), glyceraldehyde-3-phosphate dehydrogenase (GAPDH; Santa Cruz Biotechnology, sc-32233), Lamin B (Santa Cruz Biotechnology, sc-6217), E12/E47 (Santa Cruz Biotechnology, sc-365261), c-Myc (Thermo Fisher Scientific, 13-2500), hemagglutinin A (HA; Thermo Fisher Scientific, 71-5500), Histone H3 (Abcam, ab1791), and actin (EMD Millipore, MAB1501). Bound primary antibodies were detected using horseradish peroxidase-conjugated anti-mouse or -rabbit IgG (Sigma-Aldrich, A9044, or Thermo Fisher Scientific, G-21234, respectively) and SuperSignal ${ }^{\mathrm{TM}}$ West Pico PLUS Chemiluminescent Substrate (Thermo Fisher Scientific, 34580).

\section{Cycloheximide (CHX) chase assay}

HEK 293T cells were seeded in Dulbecco's modified Eaglés medium (DMEM) supplemented with 10\% fetal bovine serum (FBS), $100 \mathrm{units} / \mathrm{ml}$ penicillin, and $100 \mu \mathrm{g} / \mathrm{ml}$ streptomycin at a density of $10^{5}$ cells per well of a 6-well plate. One day later, PEI was used to transiently transfect cells with $3 \mu \mathrm{g}$ of each plasmid encoding the WT and mutant NeuroD1 strains. To block novel protein synthesis, CHX was added to the culture, $48 \mathrm{~h}$ after transfection, to obtain a final concentration of $100 \mu \mathrm{g} / \mathrm{ml}$. Additionally, $30 \mathrm{~min}$ prior to CHX addition, a mitogen-activated protein kinase (MAPK) kinase (MEK) inhibitor, PD 98059, was added, to yield a final concentration of $0.1 \mathrm{mg} / \mathrm{ml}$, to inhibit the ERK pathway. Thereafter, cells were harvested at 0, 30, 60, and 120 min following CHX addition, lysed in RIPA Lysis and Extraction Buffer containing the Protease Inhibitor Cocktail, and subjected to western blot analysis.

\section{Ubiquitination assay}

HEK 293 T cells $\left(10^{5}\right.$ cells per well of a 6-well plate) were transfected with $8 \mu \mathrm{g}$ of each plasmid encoding the Myc-tagged WT (c-Myc-tag method was previously described [19]) or NeuroD1 mutants and $2 \mu \mathrm{g}$ of pCHA-ubiquitin vector per $10^{6}$ cells. Fortyeight hours after transfection, ALLN, a proteasome inhibitor, was added to the culture to obtain a final concentration of $20 \mu \mathrm{M}$. Two hours later, cell lysates were prepared in phosphate-buffered saline (PBS) with 1\% Triton $^{\mathrm{TM}} \mathrm{X}-100$ (Sigma-Aldrich, T8787) and Protease Inhibitor Cocktail. A total of $500 \mu \mathrm{g}$ of protein was incubated with $2 \mu \mathrm{g}$ of anti-Myc antibody to precipitate NeuroD1 proteins. In order to precipitate the immune-complex, it was added to $20 \mu \mathrm{l}$ of Protein A Sepharose CL-4B slurry (GE Healthcare, 17-0780-01) and incubated at $4^{\circ} \mathrm{C}$ for $1 \mathrm{~h}$. Precipitates were separated on SDSpolyacrylamide gel and immunoblotted with anti-HA antibody to detect ubiquitin conjugates. To validate the input of NeuroD1 proteins, $10 \mu \mathrm{g}$ of each lysate was subjected to western blot analysis using anti-Myc antibodies.

\section{Luciferase assay}

One day prior to transfection, F11, P19, INS-1, and HeLa cells were seeded at a density of $1 \times 10^{5}$ cells per well of a 6 -well plate. Transfection was carried out with $1 \mu \mathrm{g}$ of each reporter plasmid of pGL3-NeuroD1, containing the $2.2 \mathrm{~kb}$-long flanking sequences of the translation initiation site of NeuroD1 [9], and pGL3-RIPE3, containing three copies of the rat insulin promoter E-box sequence [21]. The expression vectors encoding WT and S274 mutants (0.5 $\mu \mathrm{g}$ of each) were co-transfected with $0.2 \mu \mathrm{g}$ of pCR3.1-E47 [9] using $2 \mu \mathrm{g} / \mathrm{ml}$ PEI. Forty hours after transfection, cell lysates were harvested in $1 \times$ Passive Lysis Buffer ${ }^{\text {TM }}$ (Promega, E1941) and luciferase activity was determined using the Dual-Luciferase Reporter Assay System ${ }^{\mathrm{TM}}$ (Promega, E1960). During transfection, the total amount of DNA was kept constant by adding pcDNA3 (Invitrogen), and $0.1 \mu \mathrm{g}$ of thymidine kinase promoter-Renilla luciferase reporter plasmid (Promega) was used as an internal control to normalize transfection efficiency. Normalized luciferase activity was presented as a fold ratio with respect to the basal activity of the reporter gene in the absence of expression vectors.

\section{Neurite outgrowth analysis}

Cultures were prepared from mouse primary cortical neurons at embryonic day 16.5 (E16.5). Briefly, the embryonic brains of $\mathrm{NeuroDI}^{+/+}$and NeuroDI $1^{-/}$littermates were isolated and the surrounding meninges were removed. The cortices were minced and dissociated by incubation in Accumax solution ${ }^{\mathrm{TM}}$ (Sigma-Aldrich, A7089) at $37^{\circ} \mathrm{C}$ for $15 \mathrm{~min}$. Dissociated cells were washed and plated on poly-D-lysine-coated coverslips, placed in the wells of a 
12-well plate, at a seeding density of $1 \times 10^{5}$ cells per well. The cells were subsequently incubated in minimum essential medium alpha (MEM $\alpha$ ), supplemented with GlutaMAX ${ }^{\mathrm{TM}}, 10 \%$ (v/v) FBS, 0.6\% glucose, and $1 \%$ penicillin-streptomycin, at $37^{\circ} \mathrm{C}$ in a humidified atmosphere of $5 \% \mathrm{CO}_{2}$ and $95 \%$ ambient air for $2 \mathrm{~h}$. All culture reagents, except Accumax solution ${ }^{\mathrm{TM}}$, were obtained from Thermo Fisher Scientific. Thereafter, the medium was replaced with serum-free B27/neurobasal medium, and half of the volume was replaced with fresh medium of the same composition every other day. Lentiviral vectors coexpressing WT or S274 mutants and green fluorescent protein (GFP) were transduced into the primary cortical neurons in microfluidic chambers (Xona, XC450) or $\Phi$ $18 \mathrm{~mm}$ cover glasses. Following $12 \mathrm{~h}$ of transduction, the medium was replaced with fresh medium. Cell cultures were maintained for $4 \sim 8 \mathrm{~d}$ by replacing the medium with fresh medium every other day, after which cells were fixed with $4 \%$ paraformaldehyde. Immunocytochemistry was performed using anti-microtubuleassociated protein 2 (MAP2) (1:500 dilution; Millipore, AB2290) and anti-TurboGFP (1:500 dilution; Invitrogen, PA5-22688) antibodies. After washing cells with PBS containing 0.1\% Triton X-100 for $1 \mathrm{~h}$ at $25^{\circ} \mathrm{C}$, either Alexa Fluor 488 -conjugated goat anti-rabbit (1:500 dilution; Thermo Fisher Scientific, A-11034) or Alexa Fluor 568-conjugated goat anti-mouse (1:500 dilution; Thermo Fisher Scientific, A-11004) antibody was added to the sample. Additionally, nuclei were stained with Hoechst 33258 (1:10,000 dilution; Thermo Fisher Scientific, H3569). The coverslips were mounted with Fluoromount $-G^{\circledR}$ Mounting Medium (SouthernBiotech, 0100-01), and images were acquired using the confocal microscope (ZEISS, LSM710).

To assess neurite outgrowth, F11 cells were plated in growth medium (DMEM with 10\% FBS, 100 units/ml penicillin, and 100 $\mu \mathrm{g} / \mathrm{ml}$ streptomycin) and transiently co-transfected with lentiviral plasmids encoding the WT or S274 mutants, together with GFP, using PEI. Twenty-four hours later, transfected cells were re-plated into 6-well plates in a differentiation medium (DMEM with 0.5\% (v/v) FBS, 100 units/ml penicillin, and $100 \mu \mathrm{g} / \mathrm{ml}$ streptomycin). Neurite outgrowth was monitored daily for up to $4 \mathrm{~d}$ [22]. Fluorescent live cell images were captured without immunostaining and analyzed using the NeuronJ plugin (version 1.4.3; Biomedical Imaging Group, Rotterdam, Netherlands) for ImageJ software (version 1.52; NIH, Bethesda, MD, USA). Data are presented in terms of the 25 th, 50 th, and 75 th percentiles and standard error (S.E.) of more than 200 cells.

\section{RNA sequencing analysis}

Three biological replicates of NeuroDI ${ }^{+/+}$and $\mathrm{NeuroDI}^{-/}$RNA sets were derived from mouse forebrains, including the neocortex and hippocampus, at E18. To determine differentially expressed genes among NeuroD $1^{+/+}$and NeuroD $1^{-/}$samples, preprocessed RNA-seq reads from the respective samples were aligned to the reference genome and quantified for each gene expression. To determine the potential roles of these differentially expressed genes, data from the statistical evaluation of labeled gene sets were represented in the form of volcano plots, overlapped with functional categories (including biological processes of Gene Ontology (GO) and the KEGG pathway), and profiled using g:Profiler ver. 0.6.7 [23]. The association between labeled genes and reference genes were analyzed using GeneMANIA software [24]. The gene list derived from RNA-seq analysis comparing $\mathrm{NeuroDI}^{+/+}$and $\mathrm{Neu}$ $r o D 1^{-1}$ is provided in Supplementary File 1.

\section{Quantitative RT-PCR}

Total RNA was extracted from cultured cells or E18 mouse forebrain using the Hybrid- $\mathrm{R}^{\mathrm{TM}}$ RNA extraction kit (GeneAll, 305101) and reverse-transcribed with SuperScript ${ }^{\mathrm{TM}}$ III First-Strand Synthesis System (Invitrogen, 18080051). The 1st cDNA was amplified by polymerase chain reaction (PCR) using PowerSYBR ${ }^{\circledR}$ Green PCR MasterMix (ABI, 4367659) on the StepOnePlus RealTime PCR System (ABI). Mouse GAPDH primers were used for the normalization of RNA expression. The sequences of all primers used in this study are provided in Table 1.

\section{Statistical analysis}

All data are presented as mean \pm standard error (S.E.). All statistical analyses were performed using SigmaPlot ${ }^{\mathrm{TM}}$ v14 software (Systat Software, Inc., San Jose, CA, USA). One-way ANOVA and

Table 1. qRT-PCR primer list

\begin{tabular}{lll}
\hline Genes & Forward primer sequence $\mathbf{5}^{\prime} \mathbf{- 3}$ & \multicolumn{1}{c}{ Reverse primer sequence 3'-5' } \\
Foxb1 & CTTCAAGGTGCTCAAGTCAGAC & GTTCTCGATAGCAAAGGGATGC \\
Gata3 & CTCGGCCATTCGTACATGGAA & GGATACCTCTGCACCGTAGC \\
Gbx2 & CAACTTCGACAAAGCCGAGG & ACTCGTCTTTCCCTTGCCCT \\
Lhx1 & CCCATCCTGGACCGTTTCC & CGCTTGGAGAGATGCCCTG \\
Nefh & AGACCCCCGTCAAGGAAGG & CTTCTCAGGGGATTTCGCCT \\
Pou4f2 & TGGACATCGTCTCCCAGAGTA & GTGTTCATGGTGTGGTAAGTGG \\
Slitrk6 & AGGCTCTTGCGACACTCTTTG & GTGGCACACTGATTTGGGATAAT \\
\hline
\end{tabular}


Holm-Sidak or Dunn's tests were used, when deemed appropriate, to compare differences between the various groups. When necessary, the t-test and Mann-Whitney $U$ test were also performed. To assess the normality of data, the Shapiro-Wilk test $(\mathrm{p}<0.05)$ was used.

\section{RESULTS}

\section{Deletion of NeuroD1 reduced the neurite developmental genes}

To investigate the role of NeuroD1 in differentiated neurons, we
A

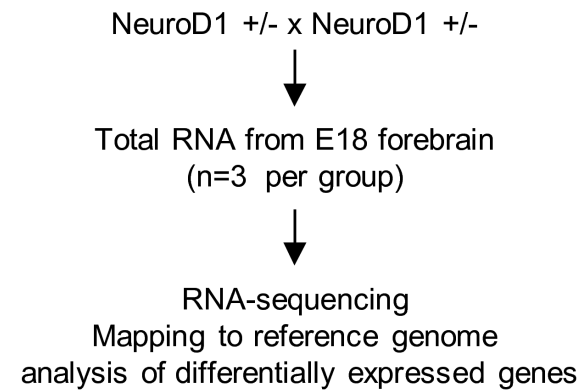

C

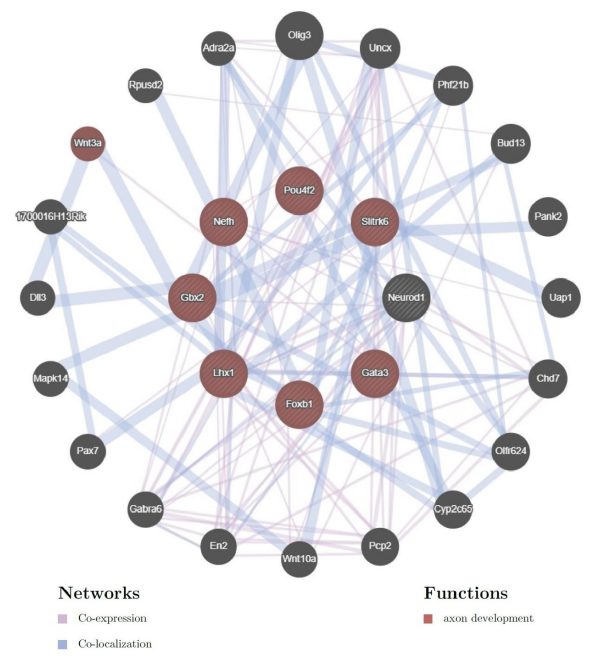

D

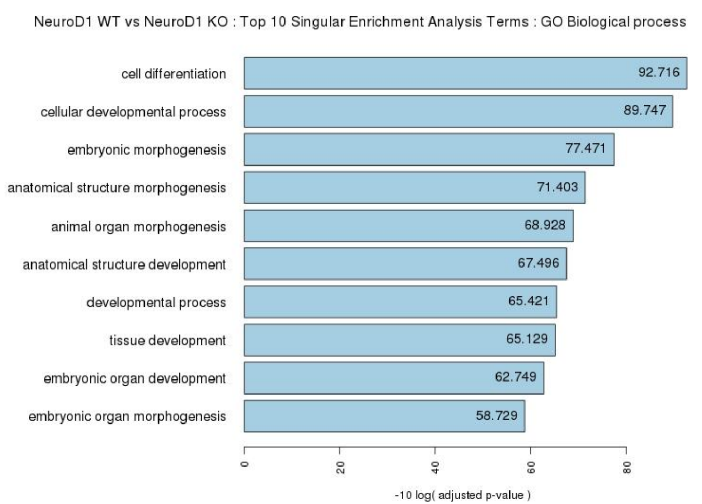

B

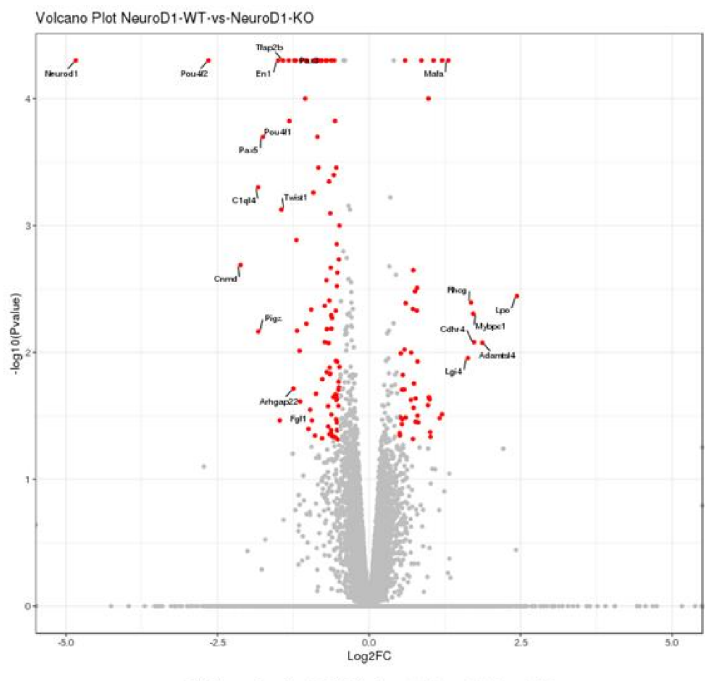

Significance (p-value S0.05, Fold-changez1.4) - FalsE + TRUE

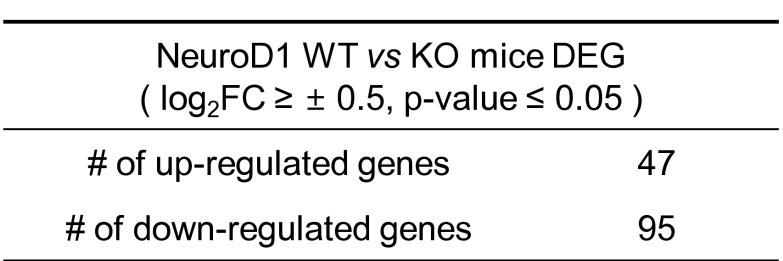

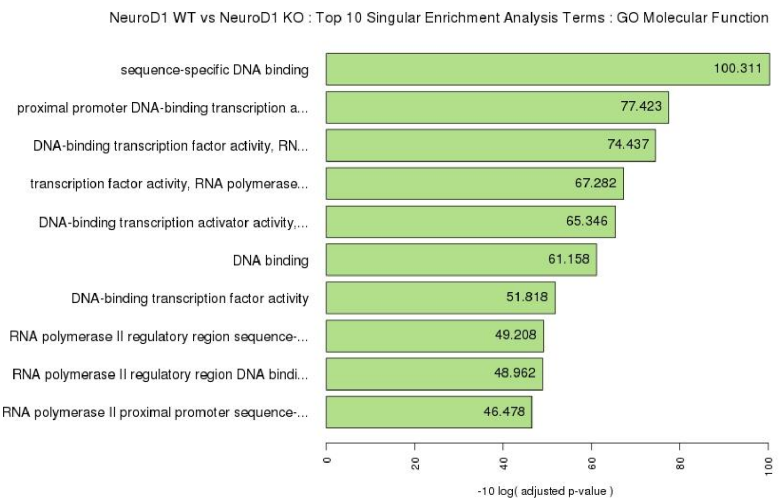

Fig. 1. RNA-sequencing analysis of isolated primary neurons from neurogenic differentiation 1 (NeuroD1) knockout (KO) mouse embryos at E18.5. (A) Scheme of the preparation of RNA for RNA-sequencing from primary neurons isolated from the NeuroD1 KO mouse forebrain. (B) Volcano plot analysis of differentially expressed genes (DEG). The number of up and downregulated genes are shown in the Table (Log2FC $\geq 0.5$; $\mathrm{p}$-value $\leq 0.05$; for 3 animals per group). (C) The network of downregulated genes, identified by GeneMANIA software, evidencing seven key genes that are related to the function of NeuroD1 regarding axon development. Gray lines connect genes that are co-localized, and purple lines indicate co-expression. (D) TOP 10 rank of the Gene Ontology (GO) biological process and molecular functions. (E) Downregulation of the seven genes were validated using real-time quantitative PCR (RT-qPCR). (F) Protein to protein interaction analysis about NeuroD1 and axon development-related genes using STRING web software. 
E

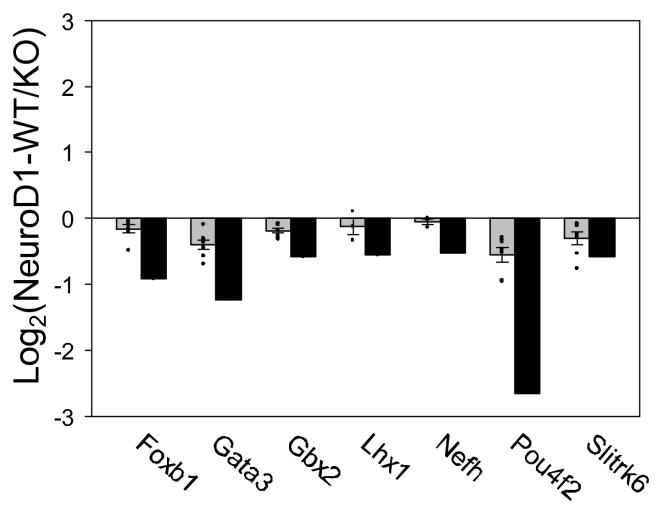

Fig. 1. Continued.

assessed the RNA profiles at gestational age 18d (E18), the latest period to consistently obtain the NeuroD1 knockout (KO) brains. RNA was extracted from the forebrains, including regions of the neocortex and hippocampus, of E18 NeuroD1 KO and WT littermates (Fig. 1A). Differentially expressed genes between $\mathrm{KO}$ and WT mice are represented as red dots and labelled in the volcano plot. A total of 47 genes were upregulated and 95 genes were downregulated (Log2 Fold Change (FC) cutoff value $\geq 0.5$ and $p$ value $\leq 0.05$ ) (Fig. 1B). A detailed gene list is provided in Supplementary File 1.

Gene Ontology (GO) enrichment analysis revealed that downregulated genes were enriched in biological processes and molecular functions for cellular development and promoter binding/ transcription, respectively (Fig. 1D). Since NeuroD1 is exclusively expressed in the nervous tissue during embryonic development, the data suggests that NeuroD1 deletion impaired neuronal transcription activity and neuronal development/differentiation. We further analyzed the relationship among the 95 downregulated genes using GeneMANIA web software [24] to delineate NeuroD1 functions specific for axon and telencephalic development. Seven genes, known to be co-expressed and co-localized with NeuroD1 (Fig. 1C), were detected, namely Gata3, Gbx2, Foxb1, Lhx1, Nefh, Pou4f2, and Slitrk6. The downregulation of these genes, triggered by NeuroD1 deletion, was further validated by quantitative reverse transcription polymerase chain reaction (qRT-PCR) analysis of RNA isolated from the embryonic forebrain using (Fig. 1E). Protein network analysis using STRING database revealed that six of the abovementioned proteins, excluding Slitrk6, interacted with NeuroD1 [25] (Fig. 1F). Gata3, Gbx2, Foxb1, Lhx1, and Pou4f2 are transcription factors that regulate neuronal differentiation in a diverse region of the brain during development and promote neurite
$\mathbf{F}$

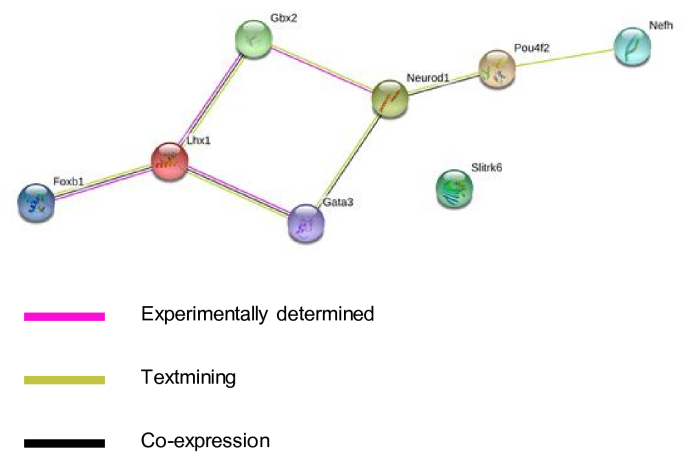

outgrowth. Nefh and Slitrk6 are structural protein that regulates axonal elongation and stabilization (see the Discussion). Our finding of potential targets that regulate neurite outgrowth suggests that NeuroD1 may be a crucial factor during brain development, particularly for neuritogenesis.

\section{Deletion of NeuroD1 reduces neurite outgrowth}

The neurite outgrowth in KO and WT primarily cultured neurons was assessed to investigate the role of NeuroD1 in differentiated neurons. An equal number of primary cortical neurons prepared from the forebrains of $\mathrm{KO}$ and WT embryos at E16.5 was plated in the cell body compartment of microfluidic chambers. At the 8th day in vitro (DIV), cells were fixed and stained for MAP2, a dendritic marker. Neurites extended through the $450 \mu \mathrm{m}$-wide microgrooves to reach the neurite compartment (Fig. 2A). Although NeuroD1-KO cortical neurons extended neurites, neurite length in the neurite compartment was significantly shorter than that of WT cortical neurons $(\mathrm{p}<0.005)$. The number of cells in the cell body compartment was similar between $\mathrm{KO}$ and WT cultures, suggesting that NeuroD1 does not affect the survival of differentiated neurons during short-term culture (Fig. 2B and C). These results indicate that NeuroD1 promotes neuron-specific functions, such as neurite outgrowth; thus, neurite outgrowth is delayed in the absence of NeuroD1.

\section{ERK regulates NeuroD1 protein stability}

The effects of ERK on the functioning of NeuroD1 are distinct for insulinoma cell lines and Xenopus embryos in a contextdependent manner $[18,19]$. Non-phosphorylated forms of S266A and S274A resulted in the accumulation of $x$ NeuroD protein in the injected Xenopus embryos. Before investigating whether ERK 
A

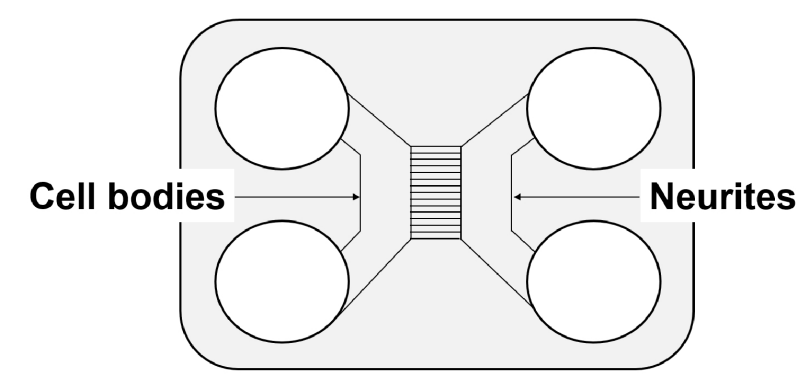

B

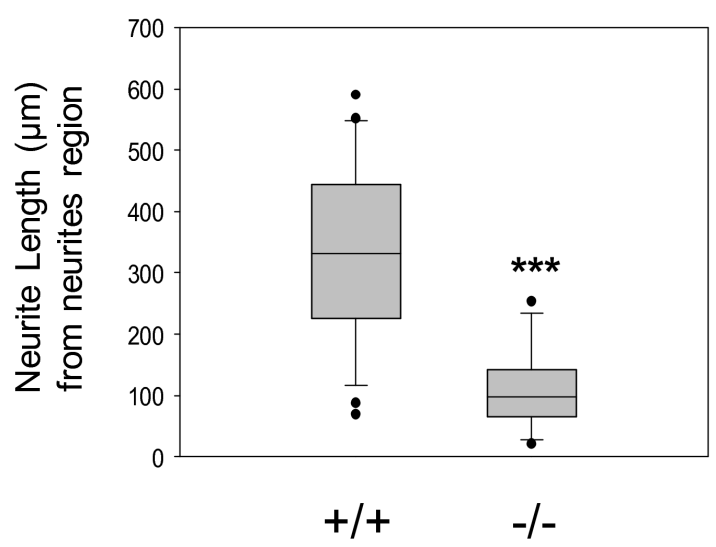

C

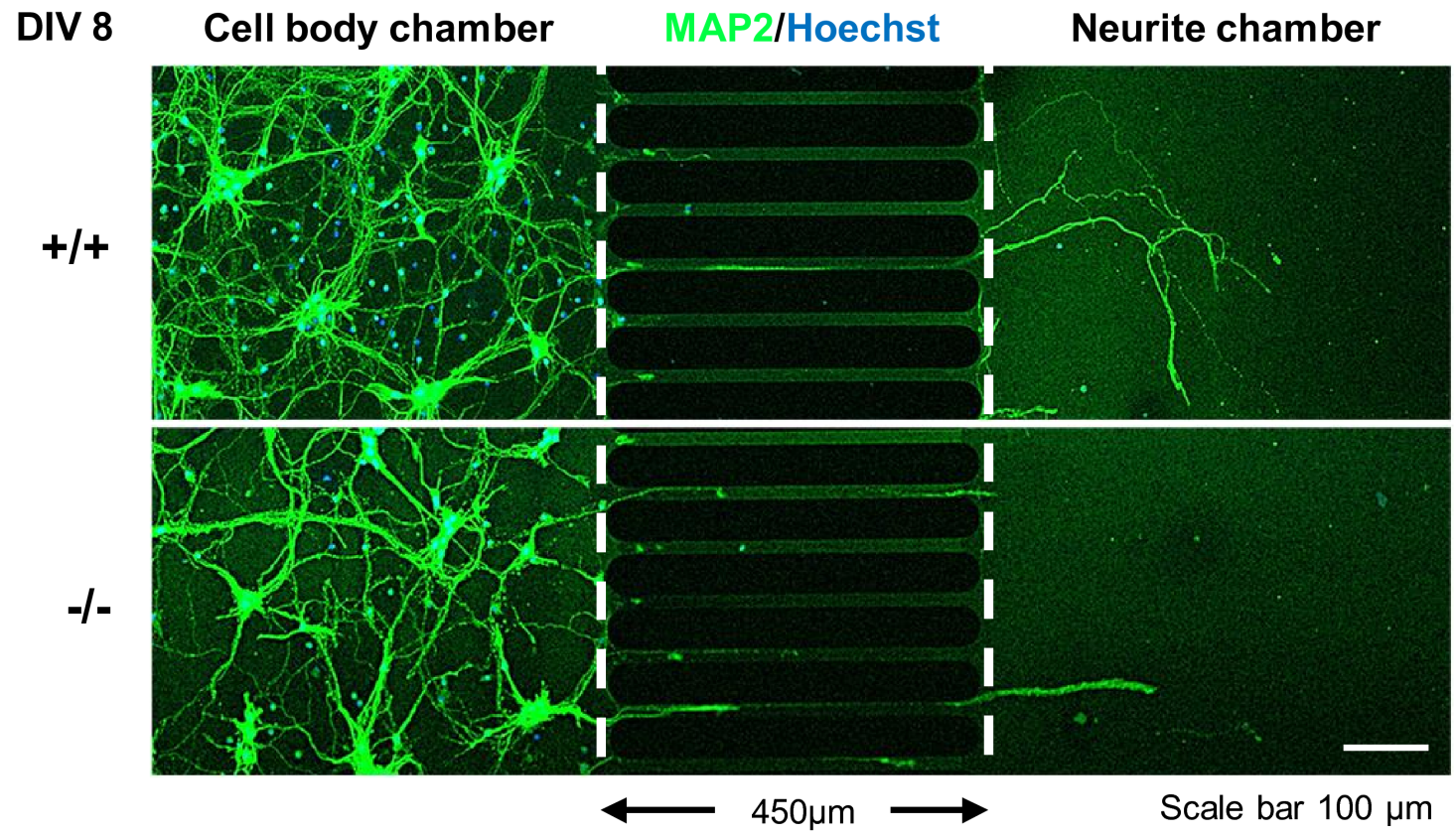

Fig. 2. Neurite outgrowth deficient in NeuroD1 knockout mouse cortical neurons. (A) Representative structure of microfluidic chambers with primary cortical neurons, isolated from the brain of NeuroD1 wild type (WT) and KO E16 embryos, plated in the cell body compartment (left). (B) Neurite lengths in the neurite compartment (right) separated by microgrooves ( $450 \mu \mathrm{m}$ long, $10 \mu \mathrm{m}$ wide) were measured at the 8 th day in vitro (DIV). (C) Cortical neurons, at 8 DIV, were immunostained by microtubule-associated protein 2 (MAP2) (green) for dendrites and Hoechst (blue) for nuclei. Data are represented as means \pm S.E. from $27 \mathrm{WT}$ and $21 \mathrm{KO}$ neurons $\left({ }^{* * *} \mathrm{p}<0.001\right.$, compared to the value of WT).

modifies the ability of NeuroD1 to promote neurite outgrowth, we first determined the effect of ERK-dependent phosphorylation on NeuroD1 protein stability using CHX chase assays. The addition of PD98059 to transfected cells, to suppress ERK-dependent phosphorylation, increased NeuroD1 protein stability (Fig. 3A and B). Among three potential serine residues (i.e., S259, S266, and S274), the Ser to Ala mutation at S274 (S274A) profoundly reduced the protein decay rate following CHX treatment (Fig. 3C E). Mutations at the other two sites, S259A or S266A, did not reduce the decay rate. These results suggest that S274 is the predominant site for ERK-dependent phosphorylation. Interestingly, the addition of ALLN, a proteasome inhibitor, dramatically increased the stability of the WT and all the mutant proteins, suggesting that NeuroD1 is a potential substrate of ubiquitination.

Following ubiquitination assay, multiple ubiquitin chains were identified in NeuroD1-containing precipitates, which were partially diminished by PD98059 (Fig. 3F). Importantly, the polyubiquitin chain entirely disappeared in the S274A mutant, whereas some chains remained in S259A or S266A mutants (Fig. 3G). These results indicated that NeuroD1 is a target of ubiquitin- 
A

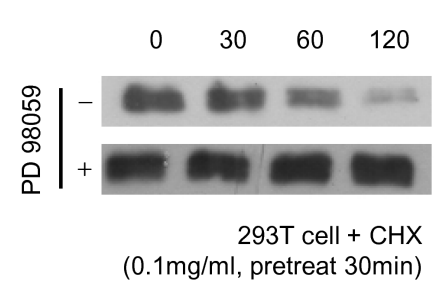

B

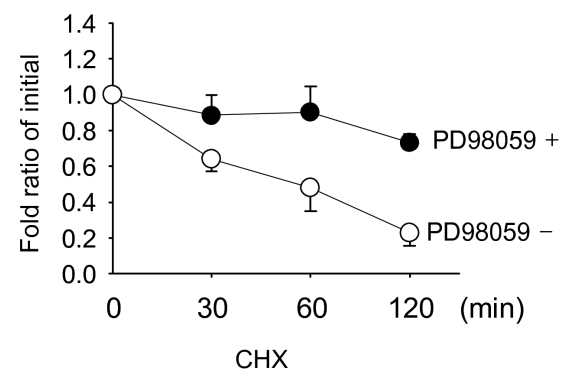

D

E

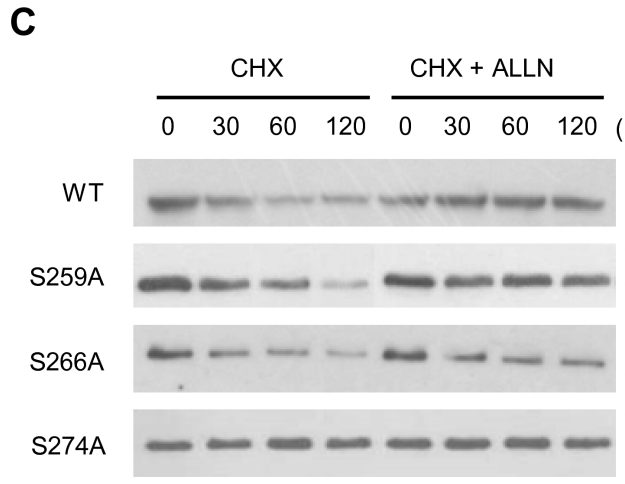

$\mathbf{F}$
G

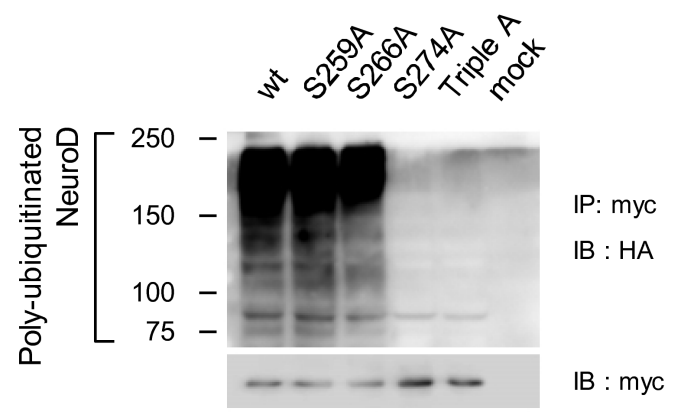

Fig. 3. Extracellular signal-regulated kinase (ERK) pathway regulates the neurogenic differentiation 1 (NeuroD1) protein stability via ubiquitination. (A) Western blot analysis of NeuroD1 protein in the absence and presence of PD 98059, a mitogen-activated protein kinase (MEK) inhibitor. Cycloheximide (CHX) was added 30 min prior to the addition of PD 98059. (B) The NeuroD1 protein intensity shown in (a) was presented with respect to the value at $\mathrm{t}=0(\mathrm{n}=3)$. (C) Western blot analysis of Myc-tagged mutants of NeuroD1 protein at ERK phosphorylation sites with and without N-acetyl-LeuLeu-norleucinal (ALLN), a proteasome inhibitor. CHX was added $30 \mathrm{~min}$ prior to adding ALLN. (D and E) NeuroD1 protein intensity shown in (c) was presented with respect to the value at $\mathrm{t}=0(\mathrm{n}=3)$. Note that the $\mathrm{S} 274 \mathrm{~A}$ mutant exhibited the highest half-life and ALLN increased the half-lives of the wild type (WT) as well as all mutants. (F) Western blot analysis showing the ubiquitination of NeuroD1 WT in the presence of ALLN. PD 98059 partially attenuated ubiquitination. (G) Myc-tagged mutants of S259A, S266A, S274A, or Triple-A were analyzed for ubiquitination. The experiments were repeated 3 times and the most representative results are shown in Fig. 3A, C, F and G. Note that S274A abolished ubiquitination, whereas S266A showed a lower level of ubiquitination compared to the WT.

proteasome-dependent degradation induced by ERK-dependent phosphorylation at S274.

\section{NeuroD1 nuclear localization is reduced in the S274D mu-} tant

A previous study showed that S274 mutation affects nuclear localization in insulinoma cell lines [18]. We tested whether the ef- 
fects of ERK-dependent phosphorylation are reproduced equally in cells other than those of insulinoma cell lines. We transfected HEK 293T cells with expression vectors for WT and mutants, and the cell lysate was fractionated to isolate three compartments: cytosolic, nuclear, and chromatin-bound extracts. Subcellular fractionation methods were validated by the presence of GAPDH (cytosol), Lamin B (nucleus), and Histone H3 (chromatin-bound) (Fig. 4A). The WT and S274A mutant were expressed in both the cytosol and nucleus, which were expressed at higher levels in the nucleus. This tendency was more pronounced with regards to the S274A mutant (Fig. 4B). In contrast, S274D is mostly expressed in the cytosol but barely present in the nuclear fraction. Immunos- taining with an anti-Flag antibody revealed strong signals for WT and S274A in the nucleus, but weak signals for S274D (Fig. 4C and D). These results suggest that the nuclear translocation of NeuroD1 is retarded following ERK-phosphorylation.

To determine whether S274 mutations alter the ability of NeuroD1 proteins to heterodimerize with class A protein families, such as E47, coimmunoprecipitation assays were performed. HEK 293T cells were co-transfected with expression vectors for the WT and mutant NeuroD1, together with E47. Since both NeuroD1 and E47 independently contain nuclear localization signals and form heterodimers in the nucleus, we used cytosolic fractions for coimmunoprecipitation. The immunoprecipitates containing Flag-
A

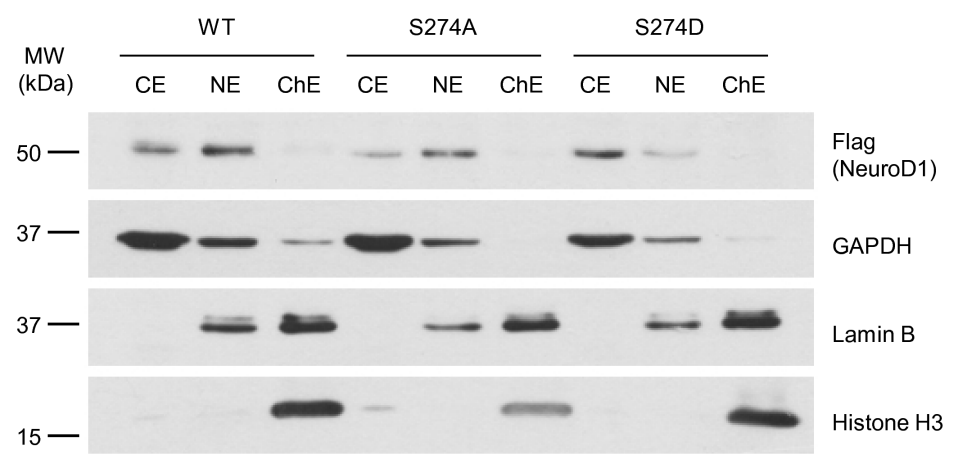

C

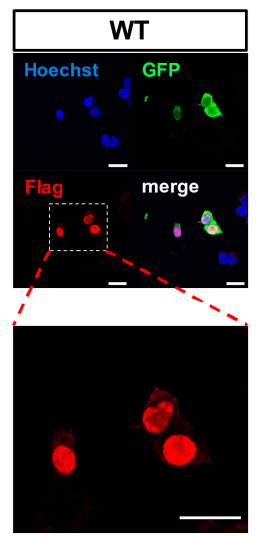

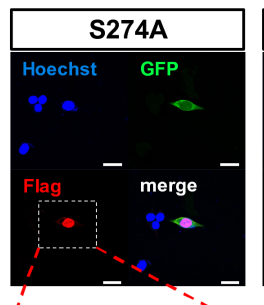

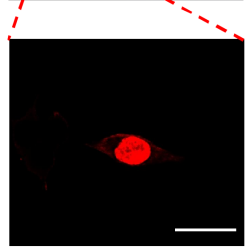

S274D
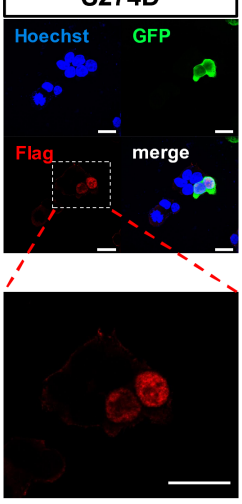

Scale bar $20 \mu \mathrm{m}$
B

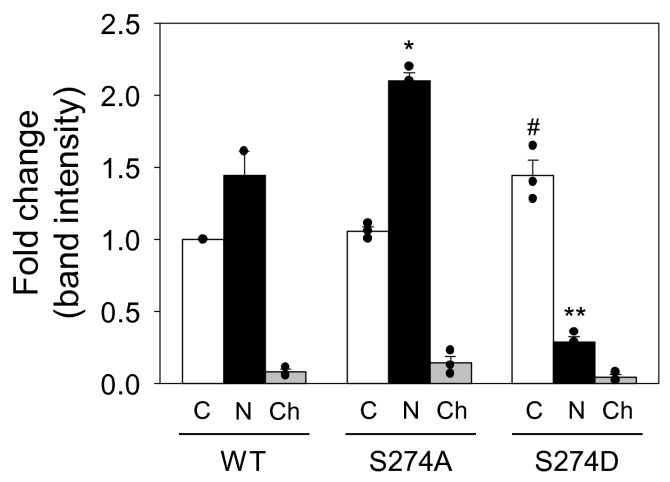

Fig. 4. Subcellular localization and heterodimer formation with E47. (A) Flag-tagged neurogenic differentiation 1 (NeuroD1) wild type (WT) and mutants were expressed in HEK 293T cells and subcellular proteins were fractionated. Glyceraldehyde 3-phosphate dehydrogenase (GAPDH), LaminB, or Histone $\mathrm{H} 3$ were used as internal controls for the cytoplasmic extract (CE), nuclear extract (NE), and chromatin bound extract (ChE). (B) Data from (a) are represented as means \pm S.E. from 3 independent experiments $\left({ }^{*} \mathrm{p}<0.05\right.$, compared to the value of the WT CE; ${ }^{*} \mathrm{p}<0.05$; ${ }^{* *} \mathrm{p}<0.01$, compared to the value of WT NE). (C) Immunocytochemistry showing Flag-tagged WT, S274A, and S274D in HEK 293T cells. Flag (red); green fluorescent protein (GFP) (green); Hoechst (blue) for nuclei. (D) The fluorescence intensity of Flag-tagged NeuroD1/GFP was quantified using the ImageJ software program. Data from $(c)$ are represented as means \pm S.E. with respect to the value of $W T\left({ }^{*} \mathrm{p}<0.05\right)$. (E) Co-immunoprecipitation assay was used to assess heterodimer formation of NeuroD1 and E47. Note that the WT, S274A, and S274D retain similar binding activity to E47 protein. Actin was used as a loading control. 
tagged WT, S274A, and S274D were immunoblotted to detect the presence of E47. The immunoprecipitate content of E47 was proportional to the amount of total immune-complex in the cytosol. This suggests that S274 mutations do not significantly alter the heterodimer-binding activity of NeuroD1 and E47 in the cytosol (Fig. 4E). By comparison, the NeuroD1 content in E47-containing immunoprecipitates appeared slightly higher in case of the WT than that in case of the mutants. It is not practically feasible to delineate the underlying mechanisms for this at present, as several factors may influence the coimmunoprecipitation efficiency, including relative protein amounts, E47 homodimer formation, and steric hindrance caused by other protein interactions. Importantly, S274 phosphorylation, as demonstrated by S274D, does not significantly alter the ability of NeuroD1 to form heterodimers with E47.

\section{S274 phosphorylation attenuates transactivation potential of NeuroD1}

We tested whether ERK-induced proteolysis correlated with the ability of NeuroD1 to transactivate downstream genes in neuronal cells. Previous studies evidenced ERK transactivated the C-terminus of NeuroD1 (155-355 aa) that was conjugated to the GAL4 DNA-binding domain in insulinoma cell lines, such as MIN6, $\beta$ TC3, or INS- $1[17,18]$. Unlike previous studies, we used full length (1-355 aa) NeuroD1 proteins in the neuronal cell line, F11. For this, we generated pLenti (lentivirus transfer) vectors for concomitant expression of the WT or S274 mutants together with GFP protein. Concomitant expression of GFP and Flag-tagged NeuroD1 proteins was validated by western blot analysis (Fig. 5A). Since class B bHLH transcription factors, such as NeuroD1, form heterodimers with their class A partners, such as E47 [26], transfection was performed with and without E47. A reporter gene containing rat insulin promoter element 3 (RIPE3), an E-box containing enhancer that is known to be activated by NeuroD1,

A

B

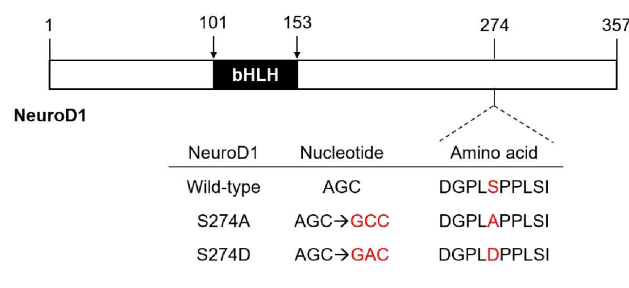

Flag-NeuroD1

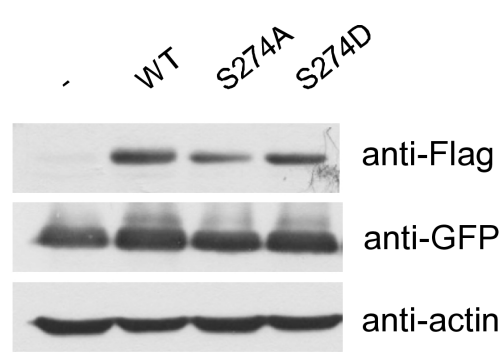

pGL3-RIPE3-TATA
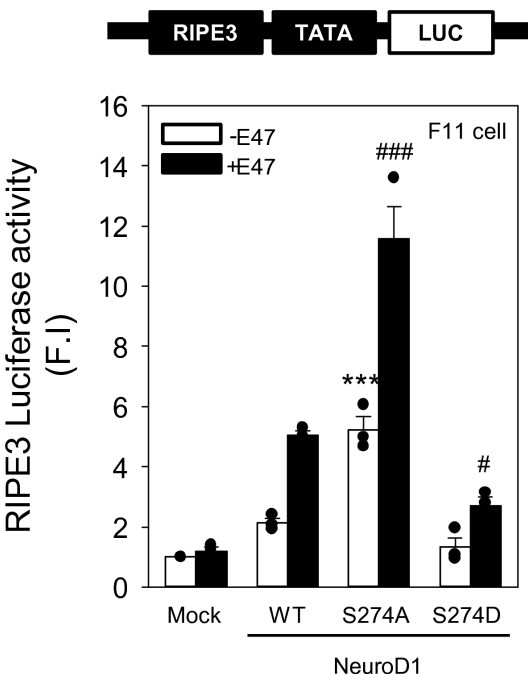

pGL3-NeuroD(-2.2kb)

NeuroD1 (-2.2kb) LUC

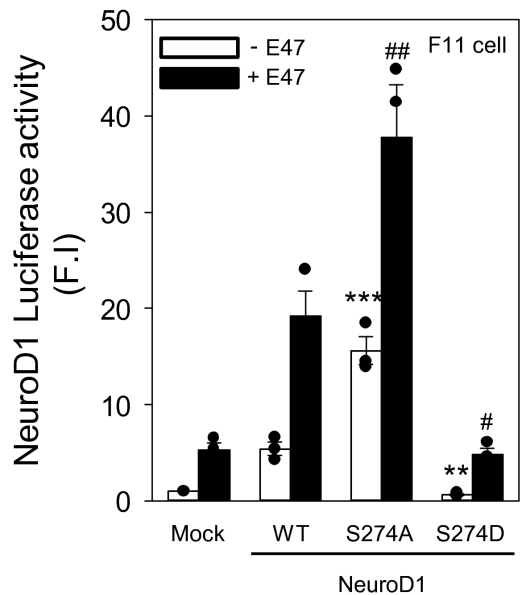

Fig. 5. Transactivation and neurite-promoting activity of neurogenic differentiation 1 (NeuroD1) wild type (WT) and mutants in F11 cells. (A) Schematic diagram of the WT and S274 mutants in the lentivirus transfer vector. Validation of both green fluorescent protein (GFP) and Flag-tagged NeuroD1 proteins by western blot analysis. (B) Schematic representation of the luciferase reporter gene of rat insulin promoter element 3 (RIPE3). Schematic representation of the $2.2 \mathrm{~kb}$ promoter region of NeuroD1 containing at least two E-box sequences. F11 cells were transfected with expression vectors for WT and mutants with and without E47. Luciferase activity was presented as the relative ratio to the value of mock transfection. Note that the stimulatory effect of full length S274A was the highest in the presence of E47, whereas the effect of the S274D mutant was minimal. Data are represented as means \pm S.E. from at least 3 independent experiments $\left({ }^{* *} \mathrm{p}<0.01 ;{ }^{* *} \mathrm{p}<0.001\right.$, compared to the value of the WT without E47; ${ }^{*} \mathrm{p}<0.05 ;{ }^{* *} \mathrm{p}<0.01 ;{ }^{* * *} \mathrm{p}<0.001, \mathrm{compared}$ to the value of the WT with E47). (C) S274A mutant NeuroD1 significantly increase transcriptional activity, S274D mutant NeuroD1 was the opposite. ${ }^{* *} \mathrm{p}<0.01,{ }^{* * *} \mathrm{p}<0.001$, compare to the value of wild-type NeuroD1, respectively. Data are means \pm SEM; $n=3$ in HeLa and P19, $n=11$ in INS-1, $\mathrm{n}=\mathrm{number}$ of independent cell culture preparations. (D) F11 cells were transfected with lentiviral transfer vectors encoding GFP with WT or S274 mutants. Following transfection, fluorescence images were obtained at the indicated time post-transfection. (E) Neurite length was quantified by the NeuronJ plugin of the ImageJ software program. Data are represented as means \pm S.E. from at least 50 transfected cells per group ${ }^{* * *} \mathrm{p}<0.001$, between groups). 
C

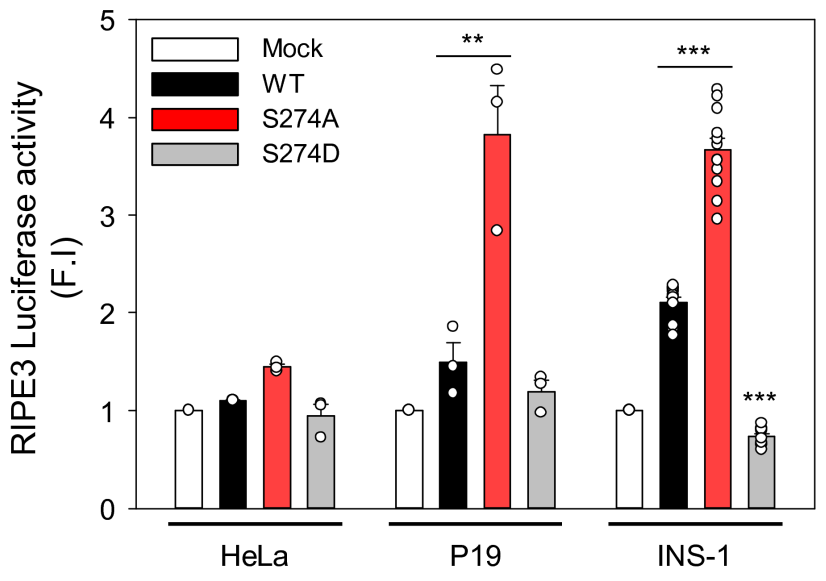

D

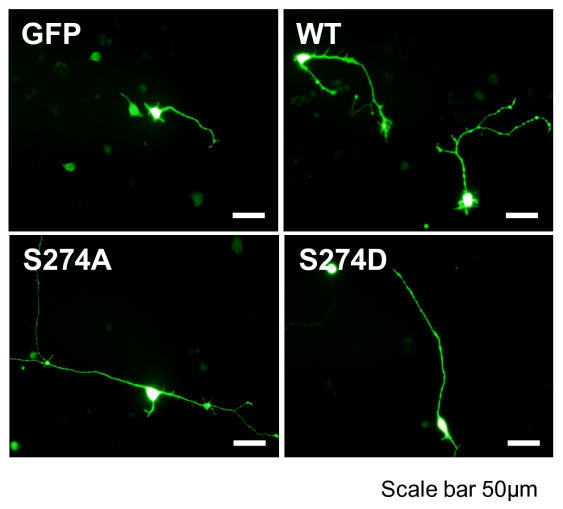

\section{E}

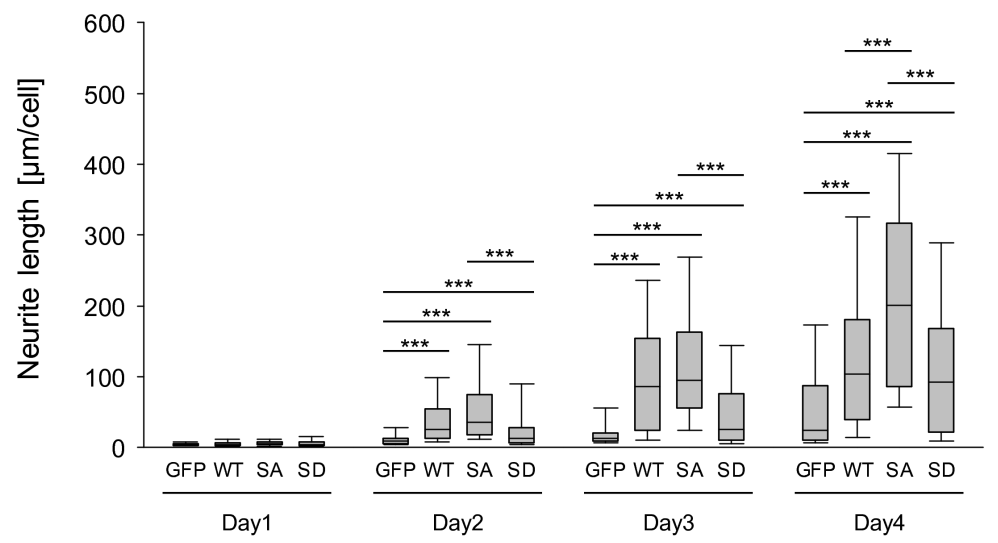

Fig. 5. Continued.

was used $[9,12]$. In contrast to that of insulinoma cell lines, the S274A mutant activated RIPE3, containing the reporter gene, by 5-fold, which further increased to 11-fold when transfection was performed with E47. In contrast, S274D, a non-phosphorylated mutant, only activated RIPE3 by 2 -fold, even when transfected with E47. To further verify the NeuroD's functions, a reporter gene containing the 2.2-kb promoter region of NeuroD1 [9] was used as it was evidenced to be autoregulated by NeuroD1 through Ebox sequences [27]. The $2.2 \mathrm{~kb}$ fragment of NeuroD1 promoter was also activated by S274A by 40 - and 18 -fold with and without E47, respectively (Fig. 5B). NeuroD1-mediated gene activation is cell-type specific. S274A enhanced the expression of both reporter genes in pluripotent P19 cells, or INS-1 insulinoma cells, and in F11 cells but not in HeLa cells (Fig. 5C). Importantly, the full length NeuroD1 can transactivate the E-box containing promoter in both F11 neuronal and INS-1 insulinoma cells. These results clearly indicate that ERK-dependent phosphorylation at S274 exerts negative effects on the NeuroD1 protein.

We used F11 cells to investigate whether increased NeuroD1 activity promotes neurite outgrowth in differentiated neurons. F11 cells provide an in vitro system for assessing the proneural functions of NeuroD1 and Neurogenin 1 (Ngn1), which can be observed through neurite outgrowth [22]. F11 cells were transfected with pLenti-plasmids encoding NeuroD1 proteins together with GFP, and neurite outgrowth was assessed in GFP-expressing cells. In the presence of low serum levels, neurite outgrowth occurred slowly in F11 cells with the GFP control vector and were only evident on day 4 (Fig. 5D). Overexpression of WT and S274A mutant accelerated neurite outgrowth significantly from day 2 , and neurite length was maximal at day 4. Moreover, the stimulatory effect was higher with S274A than the WT. By comparison, overexpression of S274D caused a delay in neurite outgrowth on days 2 and 3, but 
reached a level similar to that of the WT on day 4 (Fig. 5E). These results suggest that transactivation activity of NeuroD1, demonstrated by S274A and S274D, correlates with the transactivation function of NeuroD1 in mature neurons.

\section{WT and S274A rescue neurite outgrowth in NeuroD1 KO neurons}

To determine whether the stimulatory effect of NeuroD1 observed in F11 neuronal cells is also conserved in primary neurons, rescue experiments were performed with the WT and mutants from primary cortical neurons isolated from NeuroD1 KO embryos. On day 4, total and longest neurite lengths were reduced in $\mathrm{KO}$ neurons compared to those of the WT, indicating that our approach of using a 4-day culture was as valid as the 8-day long microfluidic chamber assay in terms of dissociated cultures. Several types of lentiviral vectors, encoding GFP in conjunction with WT or mutant NeuroD1 proteins, were transduced to KO neurons to assess the effect of ERK-dependent phosphorylation (Fig. $6 \mathrm{~A} \sim \mathrm{C})$. Expression of the WT rescued neurite outgrowth in $\mathrm{KO}$ neurons, which was similar to the case for WT neurons $(\mathrm{p}<0.01)$. Importantly, the S274A mutant also significantly enhanced total and longest neurite lengths similar to that of WT neurons $(\mathrm{p}<0.05)$. By comparison, S274D expression could increase neurite length to a lesser degree; thus, the difference between S274D and the GFP control was statistically insignificant $(\mathrm{p}=0.295)$. The number of neurites was around 4 per cell in all experimental groups (Fig. $6 \mathrm{D} \sim \mathrm{G})$. The results suggest that ERK-dependent phosphorylation of S274 may attenuate the function of NeuroD1 in neurite extension without affecting the formation of new neurites.

\section{DISCUSSION}

NeuroD1 is a tissue-specific transcription factor and its functions are regulated by several kinases that transmit the growthand activity-related extracellular signals to the nucleus. Several studies conducted over the past decade have evidenced that transcriptional activity of NeuroD1 appears to be oppositely regulated by ERK in neurons and pancreatic $\beta$ cells. In insulinoma cell lines, ERK, triggered by glucose stimulation, appeared to activate the transactivation potential of NeuroD1, when functioning as a hybrid protein with the GAL4 DNA-binding domain. In contrast, a phosphorylation-null mutation at the ERK phosphorylation site increased proneural functions of NeuroD1 in early embryogenesis. The present study has clarified a long-standing enigma regarding the impact of ERK phosphorylation on functions of NeuroD1.

Like other members of the class B bHLH transcription factor family $[28,29]$, the NeuroD1 protein undergoes rapid decay via ubiquitin-dependent degradation (Fig. 3). The degradation rate declines when the ERK pathway is blocked with a MEK inhibitor. Ala substitution at the ERK phosphorylation site (S274A) also lowers the protein decay rate and increases the amount of protein in the nuclear compartment (Fig. 4). Unlike previous studies [17, 18] using a hybrid of NeuroD1 (156-355) with Gal4 DNA-binding domain (DBD), the current study evidenced that full-length NeuroD1 proteins are capable of upregulating the expression of downstream reporter genes and that the stimulatory effect is higher with the S274A mutation compared to the WT (Fig. 5). Similar results were obtained with full-length NeuroD1 in P19, an embryonic carcinoma cell line, and INS-1, a rat insulinoma cell line (Fig. 5C), suggesting that the ERK pathway negatively regulates the functions of NeuroD1. We summarized of the effect S274A mutation in diverse cell types in Table 2. Likewise, neurite outgrowth is accelerated with the S274A mutation than that of the WT in F11 neuronal cells (Fig. 5). The S274A mutation does not alter the innate activity of the NeuroD1 protein with regards to nuclear localization or heterodimer formation with E47 (Fig. 4). Thus, the increased activity of S274A can be explained by its high content, due to reduced decay rates or increased protein stability.

In contrast, S274D, a phosphomimetic mutant of ERK, demonstrated poor activity in transactivating reporter genes or inducing neurite outgrowth compared to that of the WT protein. Although the cytosolic content of S274D was relatively high compared to that of the WT or S274A, the nuclear localization of S274D was significantly attenuated (Fig. 4A D). Thus, the poor activity of S274D in transactivation or neurite promotion is due to the low content of functional complexes containing the S274D protein in the nuclear compartment, and consequently low occupancy of the promoter. E47 contains nuclear localization sequences at 165-169 aa and 539-554 aa; thus, it is able to direct nuclear localization of a partner protein in heterodimer complexes containing a tissuerestricted class B bHLH protein partner, such as $\mathrm{MyoD}$ [28]. Since S274D in the cytosolic compartment can interact and form a heterodimer with E47 (Fig. 4D), the low content of S274D in the nuclear compartment implies that the S274D-containing complex is prohibited from the nuclear compartment, which cannot be negated by the nuclear localization sequences of E47. Further studies are necessary to reveal whether and, if so, how ERK-dependent phosphorylation induces tethering of the heterodimer complex to cytosolic adaptor(s).

Although this study has revealed the molecular mechanism of Erk-dependent NeuroD1 regulation via observing neurite outgrowth in differentiated neuronal cells, this finding can be expanded to elaborate cell fate determination of the uncommitted stem cells. During the development of forebrain, ERK signals exert 
A

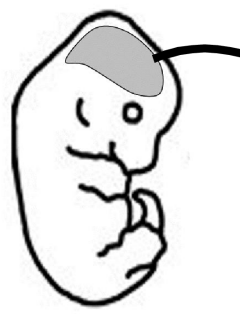

Mouse forebrain from E16.5 NeuroD1 +/+ or -/-

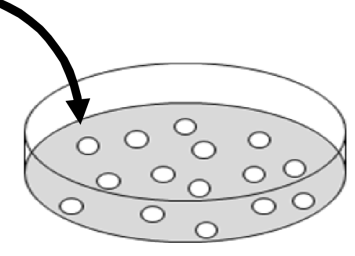

Primary cortical neuron cultures
Lentiviral vectors co-expressing

GFP and NeuroD1

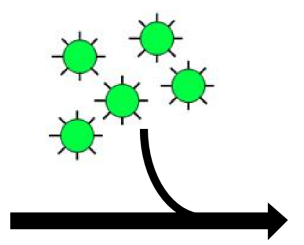

Transduction at DIV 1

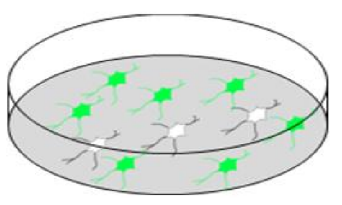

Fixation at DIV4 and immunocytochemistry

\section{B}

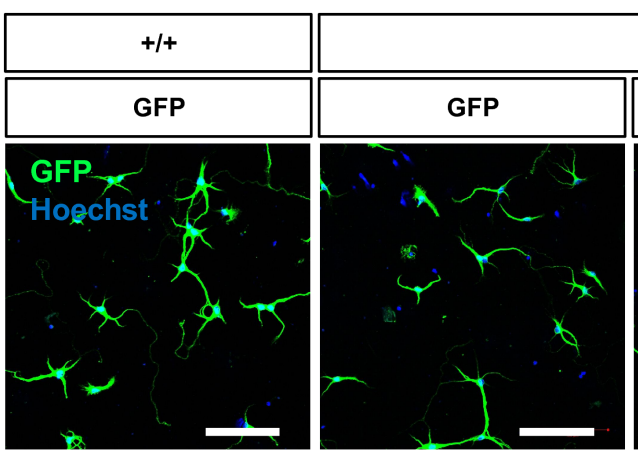

C

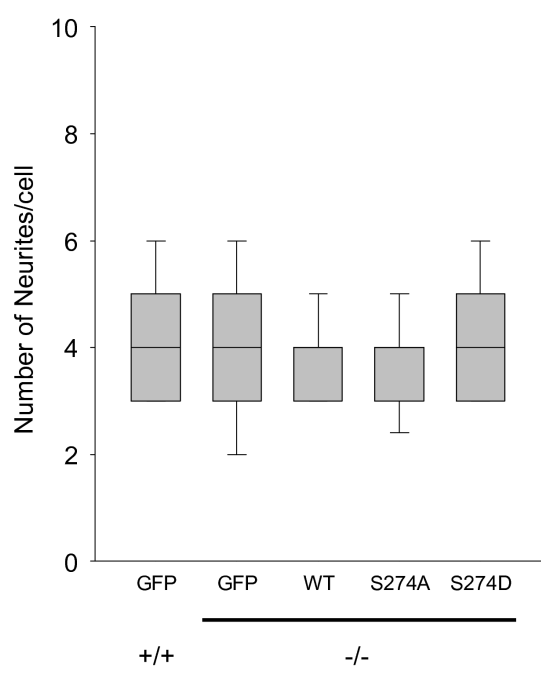

D

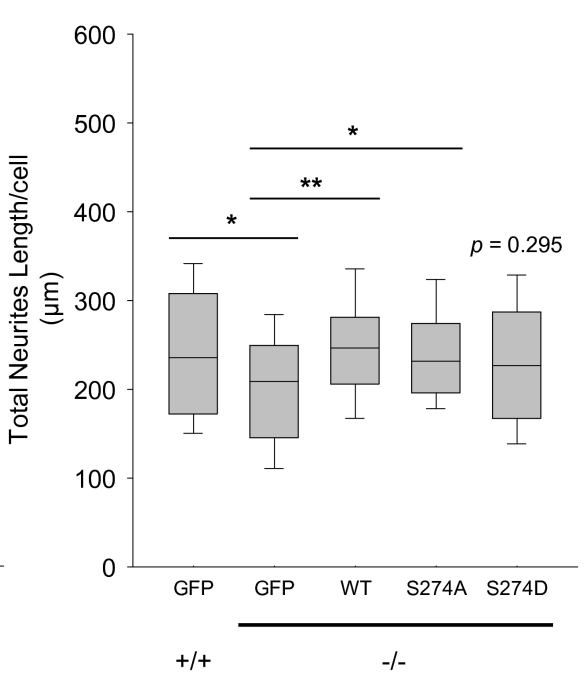

$-1-$

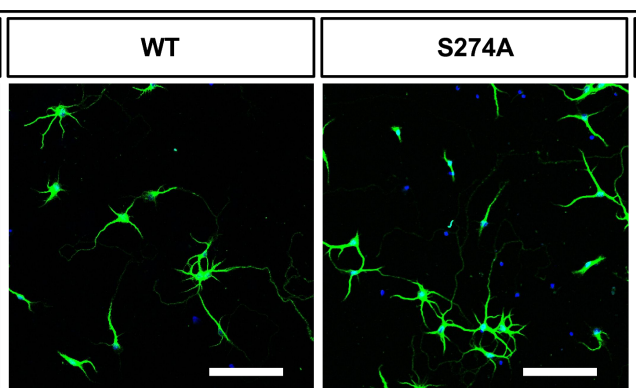

E
Scale bar $150 \mu \mathrm{m}$

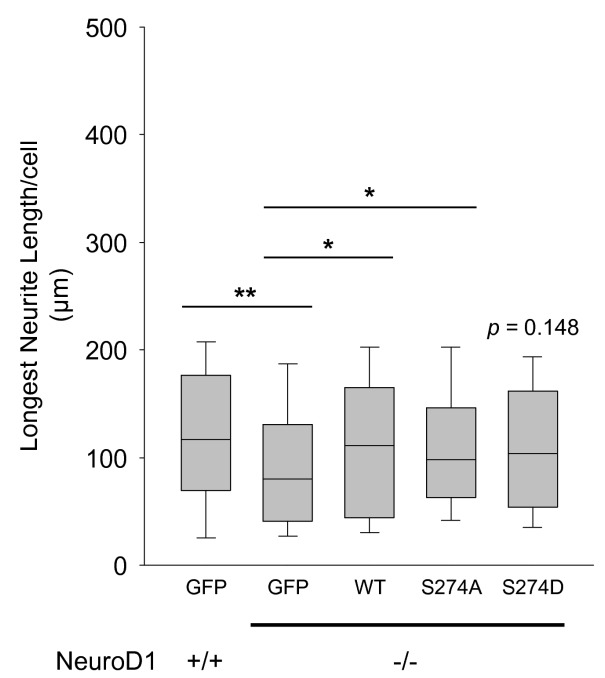

Fig. 6. Rescue experiments to supplement neurogenic differentiation 1 (NeuroD1) deficiency. (A) Schematic representation of the experimental process for transducing primary cortical neurons from the brain of NeuroD1 knockout $(\mathrm{KO})$ embryos. One day after plating, cortical neurons were transduced with lentiviral vectors encoding green fluorescent protein (GFP) together with wild type (WT) or S274 mutants. Cells were fixed at the 5th day in vitro (DIV). (B) Cells were immunostained with anti-GFP antibody (green) to detect lentivirus transduced neurons and Hoechst (blue) for nuclei. (C E) Neurite length was quantified from images shown in (B) using the NeuronJ plugin of the Image software program. The number of neurites, total neurite length, and longest neurite length per cell were counted and presented. Data are represented as means \pm S.E.; at least 50 neurons were counted per group $\left({ }^{*} \mathrm{p}<0.05 ;{ }^{* *} \mathrm{p}<0.01\right.$, compared to the value of KO neurons expressing GFP as a control).

opposite effects on neuronal and astroglial differentiation. ERK suppresses neuronal differentiation by inhibiting the expression of Neurogenins 2 (Neurog2), a proneural transcription factor, in dorsal progenitor cells during cell cycle progression. At the commitment stages of progenitor cells, transcription of Neurog2 and NeuroD1 (the target gene of Neurog2) increases as ERK signals 
Table 2. Summary of the effect of S274A mutation in diverse cell types

\begin{tabular}{|c|c|c|c|c|c|c|c|}
\hline \multirow{2}{*}{ Cell type } & \multirow{2}{*}{ Cell name } & \multirow{2}{*}{ NeuroD1 construct } & \multirow{2}{*}{$\begin{array}{l}\text { Protein length } \\
\text { (amino acid) }\end{array}$} & \multirow[t]{2}{*}{ Localization } & \multicolumn{2}{|c|}{$\begin{array}{c}\text { Transcriptional } \\
\text { analysis }\end{array}$} & \multirow{2}{*}{ References } \\
\hline & & & & & Activity & Methods & \\
\hline \multirow[t]{4}{*}{ Pancrease } & MIN6 & Gal4-DBD-NeuroD1 & $156 \sim 355$ & Cytosol>Nuclei & Low & CAT & [18] \\
\hline & $\beta \mathrm{TC} 3$ & Gal4-DBD-NeuroD1 & $156 \sim 355$ & n.a. & Low & Luciferase & [17] \\
\hline & INS-1 & Gal4-DBD-NeuroD1 & $156 \sim 355$ & n.a. & Low & Luciferase & [17] \\
\hline & INS-1 & Flag-NeuroD1 & $1 \sim 355$ & n.a. & High & Luciferase & In this study \\
\hline Cervical cancer & HeLa & Flag-NeuroD1 & $1 \sim 355$ & n.a. & High & Luciferase & In this study \\
\hline Kidney & $293 \mathrm{~T}$ & Flag-NeuroD1 & $1 \sim 355$ & Cytosol $<$ Nuclei & High & Luciferase & In this study \\
\hline \multirow[t]{2}{*}{ Neuroblastoma } & P19 & Flag-NeuroD1 & $1 \sim 355$ & n.a. & High & Luciferase & In this study \\
\hline & F11 & Flag-NeuroD1 & $1 \sim 355$ & n.a. & High & Luciferase & In this study \\
\hline
\end{tabular}

DBD, DNA Binding Domain.

decline. As a result, the cells in the dorsal forebrain exit the cell cycle and terminally differentiate into glutamatergic neurons [30]. Indeed, NeuroD1 is known to activate a cyclin-dependent kinase inhibitor, p21 and thereby induce cell cycle exit [31]. Thus, the negative regulation of Neurog2 at the transcriptional level and NeuroD1 at the post-translational level by ERK shown in the present study may demonstrate potential mechanisms for prohibiting premature cell cycle exit while maintaining cortical progenitor pools in the mid-embryonic stages. After entering late embryonic stages, the dorsal progenitor cells acquire astroglial competence and generate astrocytes at the expense of neurons [32]. Suppression of RAS-ERK pathway by deletion of Mek1/2 (also known as MAPK/ERK kinase 1/2) or deletion of ERK1/2 induces depletion of astrocytes in postnatal brain $[33,34]$, suggesting that ERK plays a role in the astroglial lineage progression of dorsal progenitor cells. Our finding of the negative regulation of NeuroD1 protein by ERK may provide a clue to elucidate the previously unexplored mechanisms for driving the genetic switch of dorsal progenitor cells from neurogenic-to-gliogenic competence after Neurog2 expression declines in the late stage of development (Fig. 7) [3537]. Impairments in lineage progression caused by malfunction of RAS-ERK signaling lead to alterations in the cortical cytoarchitecture, which is thought to represent the major underlying cause of several neurological disorders, including microcephaly or megalocephaly, and more subtle neurodevelopmental diseases, including schizophrenia, autism, epilepsy, and RASopathies [38-40].

Transcriptome analysis and GO enrichment analysis indicated that the genes involved in axonogenesis and dendrite development were downregulated in NeuroD1 KO mice (Fig. 1). In the primary neurons obtained from NeuroD1 KO embryos, neurite outgrowth is attenuated, suggesting that NeuroD1 plays a role in proneural activity regarding the development of neuronal processes. F11 cells are a hybridoma cell line between mouse neuroblastoma and rat dorsal root ganglion (pseudo-unipolar) cells and extend bipolar neurites in the presence of dibutyryl-cAMP. Without it, the F11 cells barely extend neurites up to day 3 (Fig. 5D and E), suggesting that innate neurogenic factors are limited in F11 cells. Overexpression of WT, S274D, and S274A NeuroD1 can promote neurite outgrowth in F11 cells with the highest magnitude for S274A and the lowest for S274D (Fig. 5). The data are consistent with the proteins' half-lives (Fig. 3) and transactivation potential of downstream reporter genes (Fig. 5B).

By comparison, the effect of S274A is not prominent compared to the WT in NeuroD1 -/- primary neurons (Fig. 6). It can be partly due to that axonal and dendritic morphogenesis in cortical neurons is regulated by pleiotropic factors and the loss of NeuroD1 can be compensated by other bHLH proteins, such as NeuroD2, MATH2, MATH3, and Atoh7 as shown in NeuroD1 knockout mice $[5,41,42]$. Thus, NeuroD1 -/- cortical neurons can extend neurites, although to a lesser degree, compared to WT neurons (Fig. 1 and 6). Therefore, the influence of S274A may be minimal in the rescue experiments using NeuroD1 -/- cortical neurons compared to the one in F11 cells. Importantly, S274D is less effective to rescue the NeuroDl's functions (Fig. 6), supporting our hypothesis that phosphorylation of S274 is important to determine the neuritogenic function of NeuroD1.

A previous study evidenced that NeuroD1 can be phosphorylated by an activity-induced protein kinase, CaMKII, at S336 and the S336A mutation destroyed the ability of NeuroD1 to stimulate dendritic growth in Bcl2-overexpressed cerebellar granule neurons $[43,44]$. However, similar to that of insulinoma cells [17], the transactivation activity of NeuroD1 is not altered by S336 mutations when tested with chimeric proteins fused to the Gal4 DNA-binding domain [44]. Further studies using the full length 


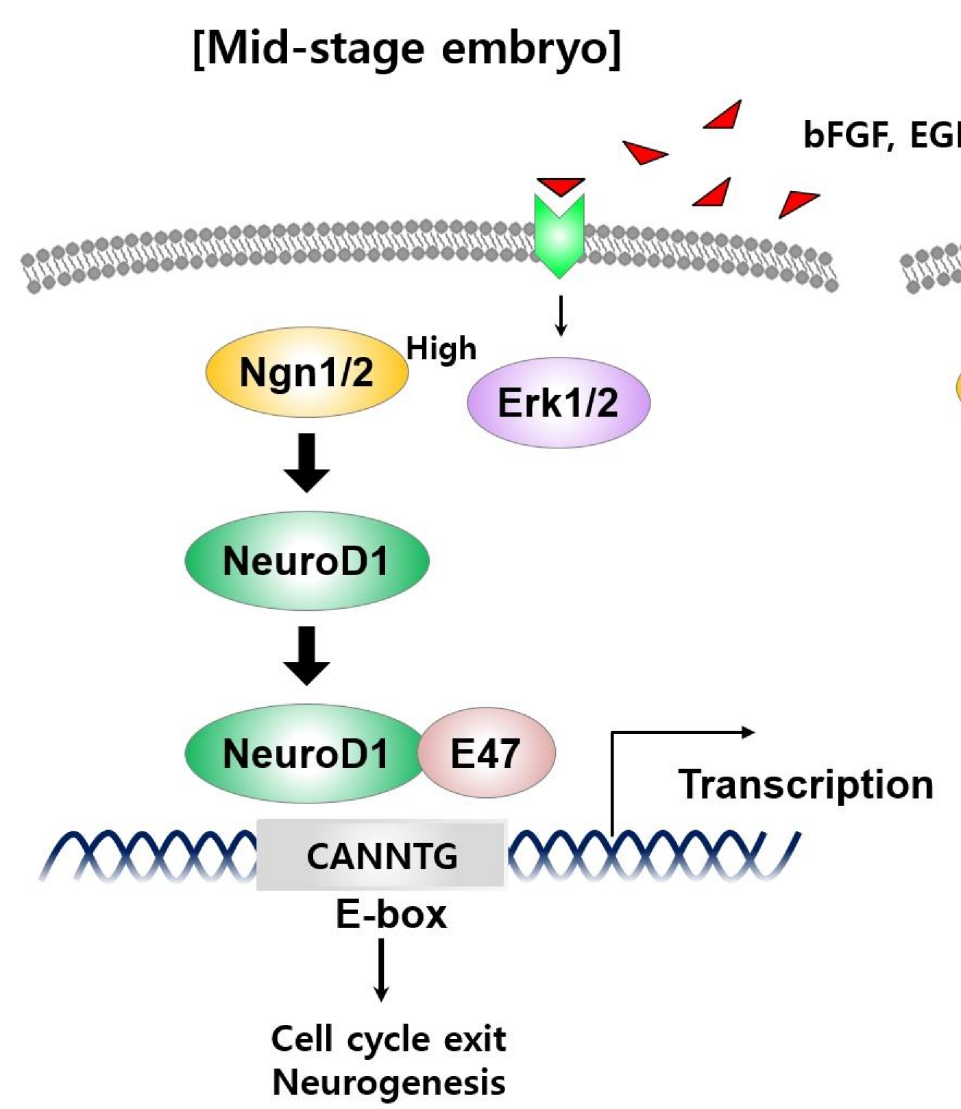

\section{[Early-, Late-stage embryo]}
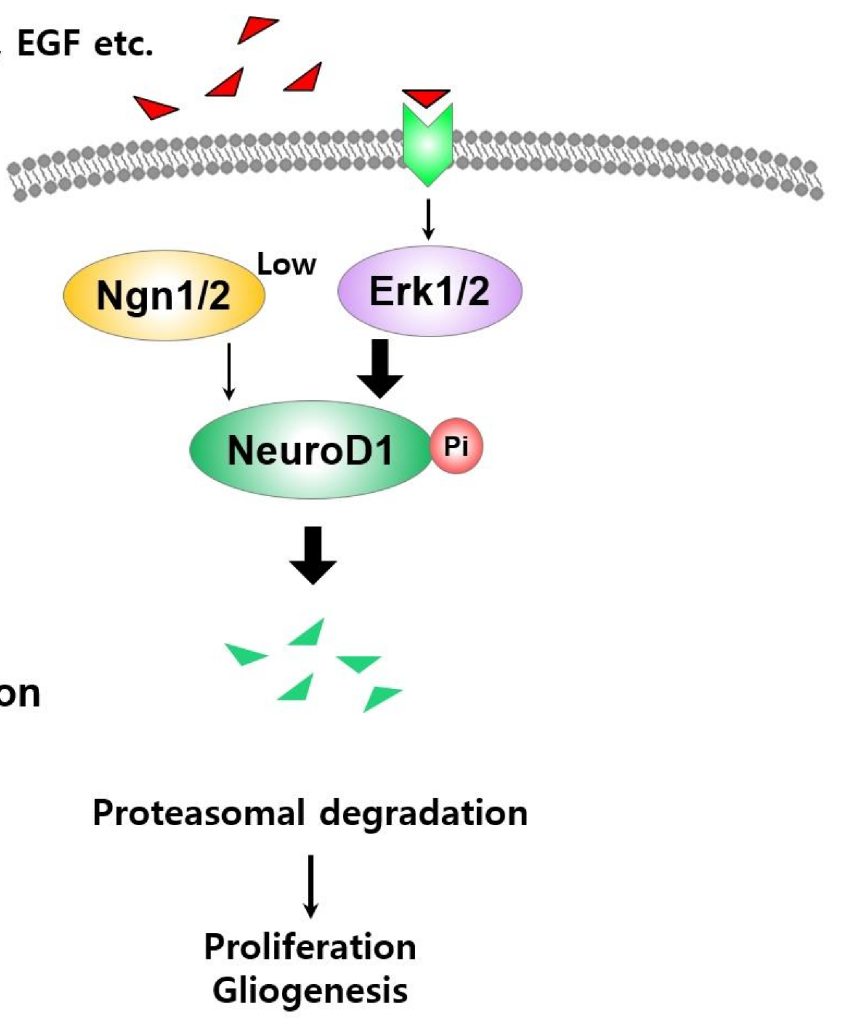

Fig. 7. Schematic model for physiological roles of ERK-dependent phosphorylation of NeuroD1. NeuroD1 proteins heterodimerize with E47, translocate to the nuclear compartment, and transactivate downstream target genes. Consequently, NeuroD1 induces cell cycle exit, neuronal differentiation, and neurite outgrowth. ERK-dependent phosphorylation at S274 facilitates ubiquitination and proteasomal degradation of NeuroD1. By negatively regulating NeuroD1, Erk maintains the progenitor pools at mid-stage brain development and promotes glial cell differentiation of progenitor cells at the expense of neurogenesis at late-stage brain development.

NeuroD1 protein may be helpful in revealing how NeuroD1 phosphorylation, triggered by CaMKII, upregulates dendritic growth in mature neurons.

The RNA sequencing analysis of 95 downregulated genes revealed the functions of NeuroD1 with respect to the GO terms of promoter binding, RNA polymerase II, and cellular developmental processes (Fig. 1D). Subsequent analysis using GeneMANIA revealed seven core genes related to the GO term of neurite outgrowth (Fig. 1). Gata3, Gbx2, Foxb1, Lhx1, and Pou4f2 are transcription factors that regulate neuronal differentiation in a diverse region of the brain during development and promote neurite outgrowth [45-49]. Nefh (Neurofilament, Heavy polypeptide) is a cytoskeletal element of axons and Slitrk6 (SLIT and NTRK like family member 6) is an integral membrane protein, both of which regulates axonal elongation and stabilization [50,51]. Interestingly, all these genes contain potential E-box sequences within $1 \mathrm{~kb}$ upstream of the transcription start site (Supplementary File 3). These 7 genes were not included in the list of previously identified target genes of NeuroD1 [52]. A possible explanation is that two different approaches were used. Previous studies adopted a gain-of-function approach by overexpressing NeuroD1 in uncommitted cells such as early Xenopus embryos or pluripotent P19 cells, whereas we adopted a loss-of-function approach with mid-stage embryonic development when neurogenesis occurs most actively. Since the NeuroDl's neurogenic function is prominent during mid-stage embryos, it would be interesting to determine whether the newly identified genes are physiologically relevant target candidates of NeuroD1 in the mammalian brain. Indeed, overexpression of S274A NeuroD1 induced the expression of Gbx2 in F11 cells on day 3, when neurite outgrowth was most prominently enhanced by $\mathrm{S} 274 \mathrm{~A}$. The expression of the other six genes was not directly affected by the overexpression of NeuroD1 proteins in F11 cells (data not shown). Gbx2 is a homeobox gene and plays a key role in patterning both vestibular and auditory components of the inner ear [53] and NeuroD1 is expressed for the proper development of sensory neurons in the inner ear $[4,54]$. Further study may reveal 
whether and how NeuroD1 and Gbx2 interact with each other and regulate inner ear development.

Recently, NeuroD1 has emerged as an important genetic therapeutic strategy to treat the diseased brain where adult neurogenesis or synaptic integration of newborn neurons are hampered. In Alzheimer's disease model (5xFAD), overexpression of NeuroD1 using a retroviral vector can induce reprogramming of reactive astroglial cells into functional neurons [55]. Similarly, overexpression of NeuroD1 also can rescue spatial memory in aged AD mice (APPxPS1) by accelerating neuronal maturation of hippocampal progenitor cells and promote functional integration of newborn neurons $[56,57]$. S274A has a longer protein half-life than WT NeuroD1, making it a better choice for overexpressing NeuroD1 to alleviate neuronal loss in a diseased brain. By blocking the ubiquitin-dependent degradation of endogenous NeuroD1 protein may provide an alternative therapeutic strategy that promotes neuronal activity by enhancing the expression of NeuroD 1's target genes.

\section{ACKNOWLEDGEMENTS}

This study was supported by grants from the National Research Foundation of Korea (NRF- 2018M3A9G1082594- to HS-K.) and the INNOPOLIS Foundation of Jeonbuk (2019-JBRD-0048-01101). We would like to thank Dr. Jacqueline E. Lee (Dep. Molecular, Cellular \& Developmental Biology, University of Colorado, Boulder, USA) and Dr. Kang Ho Chul (Dep. Physiol. Ajou University, Suwon, Korea) for providing Myc-tagged NeuroD1 and HA-tagged Ubiquitin constructs, respectively.

\section{REFERENCES}

1. Dennis DJ, Han S, Schuurmans C (2019) bHLH transcription factors in neural development, disease, and reprogramming. Brain Res 1705:48-65.

2. Lee JE, Hollenberg SM, Snider L, Turner DL, Lipnick N, Weintraub H (1995) Conversion of Xenopus ectoderm into neurons by NeuroD, a basic helix-loop-helix protein. Science 268:836-844.

3. Kim WY, Fritzsch B, Serls A, Bakel LA, Huang EJ, Reichardt LF, Barth DS, Lee JE (2001) NeuroD-null mice are deaf due to a severe loss of the inner ear sensory neurons during development. Development 128:417-426.

4. Liu M, Pereira FA, Price SD, Chu MJ, Shope C, Himes D, Eatock RA, Brownell WE, Lysakowski A, Tsai MJ (2000) Essential role of BETA2/NeuroD1 in development of the vestibular and auditory systems. Genes Dev 14:2839-2854.
5. Miyata T, Maeda T, Lee JE (1999) NeuroD is required for differentiation of the granule cells in the cerebellum and hippocampus. Genes Dev 13:1647-1652.

6. Schwab MH, Bartholomae A, Heimrich B, Feldmeyer D, Druffel-Augustin S, Goebbels S, Naya FJ, Zhao S, Frotscher M, Tsai MJ, Nave KA (2000) Neuronal basic helix-loophelix proteins (NEX and BETA2/Neuro D) regulate terminal granule cell differentiation in the hippocampus. J Neurosci 20:3714-3724.

7. Gao Z, Ure K, Ables JL, Lagace DC, Nave KA, Goebbels S, Eisch AJ, Hsieh J (2009) Neurod 1 is essential for the survival and maturation of adult-born neurons. Nat Neurosci 12:1090-1092.

8. Naya FJ, Huang HP, Qiu Y, Mutoh H, DeMayo FJ, Leiter AB, Tsai MJ (1997) Diabetes, defective pancreatic morphogenesis, and abnormal enteroendocrine differentiation in BETA2/ neuroD-deficient mice. Genes Dev 11:2323-2334.

9. Huang HP, Liu M, El-Hodiri HM, Chu K, Jamrich M, Tsai MJ (2000) Regulation of the pancreatic islet-specific gene BETA2 (neuroD) by neurogenin 3. Mol Cell Biol 20:3292-3307.

10. Schonhoff SE, Giel-Moloney M, Leiter AB (2004) Minireview: development and differentiation of gut endocrine cells. Endocrinology 145:2639-2644.

11. Mutoh H, Fung BP, Naya FJ, Tsai MJ, Nishitani J, Leiter AB (1997) The basic helix-loop-helix transcription factor BETA2/ NeuroD is expressed in mammalian enteroendocrine cells and activates secretin gene expression. Proc Natl Acad Sci U S A 94:3560-3564.

12. Naya FJ, Stellrecht CM, Tsai MJ (1995) Tissue-specific regulation of the insulin gene by a novel basic helix-loop-helix transcription factor. Genes Dev 9:1009-1019.

13. Luo H, Chen R, Yang R, Liu Y, Chen Y, Shu Y, Chen H (2014) Reprogramming of mice primary hepatocytes into insulinproducing cells by transfection with multicistronic vectors. J Diabetes Res 2014:716163.

14. Lee SH, Rhee M, Kim JW, Yoon KH (2017) Generation of insulin-expressing cells in mouse small intestine by Pdx1, MafA, and BETA2/NeuroD. Diabetes Metab J 41:405-416.

15. Ham DS, Shin J, Kim JW, Park HS, Cho JH, Yoon KH (2013) Generation of functional insulin-producing cells from neonatal porcine liver-derived cells by PDX1/VP16, BETA2/ NeuroD and MafA. PLoS One 8:e79076.

16. Song YD, Lee EJ, Yashar P, Pfaff LE, Kim SY, Jameson JL (2007) Islet cell differentiation in liver by combinatorial expression of transcription factors neurogenin-3, BETA2, and RIPE3b1. Biochem Biophys Res Commun 354:334-339.

17. Khoo S, Griffen SC, Xia Y, Baer RJ, German MS, Cobb MH 
(2003) Regulation of insulin gene transcription by ERK1 and ERK2 in pancreatic beta cells. J Biol Chem 278:32969-32977.

18. Petersen HV, Jensen JN, Stein R, Serup P (2002) Glucose induced MAPK signalling influences NeuroD1-mediated activation and nuclear localization. FEBS Lett 528:241-245.

19. Dufton C, Marcora E, Chae JH, McCullough J, Eby J, Hausburg M, Stein GH, Khoo S, Cobb MH, Lee JE (2005) Contextdependent regulation of NeuroD activity and protein accumulation. Mol Cell Neurosci 28:727-736.

20. Jiang W, Hua R, Wei M, Li C, Qiu Z, Yang X, Zhang C (2015) An optimized method for high-titer lentivirus preparations without ultracentrifugation. Sci Rep 5:13875.

21. Hwung YP, Gu YZ, Tsai MJ (1990) Cooperativity of sequence elements mediates tissue specificity of the rat insulin II gene. Mol Cell Biol 10:1784-1788.

22. Cho JH, Kwon IS, Kim S, Ghil SH, Tsai MJ, Kim YS, Lee YD, Suh-Kim H (2001) Overexpression of BETA2/NeuroD induces neurite outgrowth in F11 neuroblastoma cells. J Neurochem 77:103-109.

23. Reimand J, Arak T, Adler P, Kolberg L, Reisberg S, Peterson H, Vilo J (2016) g:Profiler-a web server for functional interpretation of gene lists (2016 update). Nucleic Acids Res 44:W83W89.

24. Montojo J, Zuberi K, Rodriguez H, Kazi F, Wright G, Donaldson SL, Morris Q, Bader GD (2010) GeneMANIA Cytoscape plugin: fast gene function predictions on the desktop. Bioinformatics 26:2927-2928.

25. Szklarczyk D, Gable AL, Lyon D, Junge A, Wyder S, HuertaCepas J, Simonovic M, Doncheva NT, Morris JH, Bork P, Jensen LJ, Mering CV (2019) STRING v11: protein-protein association networks with increased coverage, supporting functional discovery in genome-wide experimental datasets. Nucleic Acids Res 47:D607-D613.

26. Glick E, Leshkowitz D, Walker MD (2000) Transcription factor BETA2 acts cooperatively with E2A and PDX1 to activate the insulin gene promoter. J Biol Chem 275:2199-2204.

27. Fang HB, Mi Y, Zhang Y, Wu NH, Shen YF (2010) HDAC3 augments the autoregulation of neuroD gene in P19 cells. Neuroreport 21:19-23.

28. Lingbeck JM, Trausch-Azar JS, Ciechanover A, Schwartz AL (2005) E12 and E47 modulate cellular localization and proteasome-mediated degradation of MyoD and Id1. Oncogene 24:6376-6384.

29. Hindley C, Ali F, McDowell G, Cheng K, Jones A, Guillemot F, Philpott A (2012) Post-translational modification of Ngn2 differentially affects transcription of distinct targets to regulate the balance between progenitor maintenance and differ- entiation. Development 139:1718-1723.

30. Li S, Mattar P, Dixit R, Lawn SO, Wilkinson G, Kinch C, Eisenstat D, Kurrasch DM, Chan JA, Schuurmans C (2014) RAS/ERK signaling controls proneural genetic programs in cortical development and gliomagenesis. J Neurosci 34:21692190 .

31. Mutoh H, Naya FJ, Tsai MJ, Leiter AB (1998) The basic helixloop-helix protein BETA2 interacts with p300 to coordinate differentiation of secretin-expressing enteroendocrine cells. Genes Dev 12:820-830.

32. Hirabayashi $Y$, Itoh $Y$, Tabata $H$, Nakajima K, Akiyama T, Masuyama N, Gotoh Y (2004) The Wnt/beta-catenin pathway directs neuronal differentiation of cortical neural precursor cells. Development 131:2791-2801.

33. Li X, Newbern JM, Wu Y, Morgan-Smith M, Zhong J, Charron J, Snider WD (2012) MEK is a key regulator of gliogenesis in the developing brain. Neuron 75:1035-1050.

34. Imamura O, Pagès G, Pouysségur J, Endo S, Takishima K (2010) ERK1 and ERK2 are required for radial glial maintenance and cortical lamination. Genes Cells 15:1072-1088.

35. Tabata H (2015) Diverse subtypes of astrocytes and their development during corticogenesis. Front Neurosci 9:114.

36. Kriegstein A, Alvarez-Buylla A (2009) The glial nature of embryonic and adult neural stem cells. Annu Rev Neurosci 32:149-184.

37. Beattie R, Hippenmeyer S (2017) Mechanisms of radial glia progenitor cell lineage progression. FEBS Lett 591:3993-4008.

38. Kang M, Lee YS (2019) The impact of RASopathy-associated mutations on CNS development in mice and humans. Mol Brain 12:96.

39. Silbereis JC, Pochareddy S, Zhu Y, Li M, Sestan N (2016) The cellular and molecular landscapes of the developing human central nervous system. Neuron 89:248-268.

40. Desikan RS, Barkovich AJ (2016) Malformations of cortical development. Ann Neurol 80:797-810.

41. Cho JH, Tsai MJ (2006) Preferential posterior cerebellum defect in BETA2/NeuroD1 knockout mice is the result of differential expression of BETA2/NeuroD1 along anteriorposterior axis. Dev Biol 290:125-138.

42. Mao CA, Cho JH, Wang J, Gao Z, Pan P, Tsai WW, Frishman LJ, Klein WH (2013) Reprogramming amacrine and photoreceptor progenitors into retinal ganglion cells by replacing Neurod1 with Atoh7. Development 140:541-551.

43. Lee YY, Choi HJ, Lee SY, Park SY, Kang MJ, Han J, Han JS (2020) Bcl-2 overexpression induces neurite outgrowth via the Bmp4/Tbx3/NeuroD1 cascade in H19-7 cells. Cell Mol Neurobiol 40:153-166. 
44. Gaudillière B, Konishi Y, de la Iglesia N, Yao Gl, Bonni A (2004) A CaMKII-NeuroD signaling pathway specifies dendritic morphogenesis. Neuron 41:229-241.

45. Appler JM, Lu CC, Druckenbrod NR, Yu WM, Koundakjian EJ, Goodrich LV (2013) Gata3 is a critical regulator of cochlear wiring. Version 2. J Neurosci 33:3679-3691.

46. Chen L, Chatterjee M, Li JY (2010) The mouse homeobox gene $\mathrm{Gbx} 2$ is required for the development of cholinergic interneurons in the striatum. Version 2. J Neurosci 30:1482414834.

47. Lui NC, Tam WY, Gao C, Huang JD, Wang CC, Jiang L, Yung WH, Kwan KM (2017) Lhx1/5 control dendritogenesis and spine morphogenesis of Purkinje cells via regulation of Espin. Nat Commun 8:15079.

48. Wang SW, Mu X, Bowers WJ, Kim DS, Plas DJ, Crair MC, Federoff HJ, Gan L, Klein WH (2002) Brn3b/Brn3c double knockout mice reveal an unsuspected role for Brn3c in retinal ganglion cell axon outgrowth. Development 129:467-477.

49. Alvarez-Bolado G, Zhou X, Voss AK, Thomas T, Gruss P (2000) Winged helix transcription factor Foxb1 is essential for access of mammillothalamic axons to the thalamus. Development 127:1029-1038.

50. Lee S, Shea TB (2014) The high molecular weight neurofilament subunit plays an essential role in axonal outgrowth and stabilization. Biol Open 3:974-981.
51. Katayama K, Zine A, Ota M, Matsumoto Y, Inoue T, Fritzsch B, Aruga J (2009) Disorganized innervation and neuronal loss in the inner ear of Slitrk6-deficient mice. PLoS One 4:e7786.

52. Seo S, Lim JW, Yellajoshyula D, Chang LW, Kroll KL (2007) Neurogenin and NeuroD direct transcriptional targets and their regulatory enhancers. EMBO J 26:5093-5108.

53. Lin Z, Cantos R, Patente M, Wu DK (2005) Gbx2 is required for the morphogenesis of the mouse inner ear: a downstream candidate of hindbrain signaling. Development 132:23092318 .

54. Chatterjee S, Kraus P, Lufkin T (2010) A symphony of inner ear developmental control genes. BMC Genet 11:68.

55. Guo Z, Zhang L, Wu Z, Chen Y, Wang F, Chen G (2014) In vivo direct reprogramming of reactive glial cells into functional neurons after brain injury and in an Alzheimer's disease model. Cell Stem Cell 14:188-202.

56. Richetin K, Leclerc C, Toni N, Gallopin T, Pech S, Roybon L, Rampon C (2015) Genetic manipulation of adult-born hippocampal neurons rescues memory in a mouse model of Alzheimer's disease. Brain 138(Pt 2):440-455.

57. Richetin K, Moulis M, Millet A, Arràzola MS, Andraini T, Hua J, Davezac N, Roybon L, Belenguer P, Miquel MC, Rampon C (2017) Amplifying mitochondrial function rescues adult neurogenesis in a mouse model of Alzheimer's disease. Neurobiol Dis 102:113-124. 